\title{
Dirichlet Problems for some Hamilton-Jacobi Equations With Inequality Constraints
}

Jean-Pierre Aubin

Alexandre M. Bayen

Patrick Saint-Pierre

SCUOLA NORMALE SUPERIORE

PISA 


\title{
Dirichlet Problems for some Hamilton-Jacobi Equations With Inequality Constraints
}

\author{
Jean-Pierre Aubin ${ }^{1}{ }^{2}$, Alexandre M. Bayen ${ }^{3}$ and Patrick Saint-Pierre ${ }^{4}$
}

\begin{abstract}
We use viability techniques for solving Dirichlet problems with inequality constraints (obstacles) for a class of Hamilton-Jacobi equations. The hypograph of the "solution" is defined as the "capture basin" under an auxiliary control system of a target associated with the initial and boundary conditions, viable in an environment associated with the inequality constraint. From the tangential condition characterizing capture basins, we prove that this solution is the unique "upper semicontinuous" solution to the HamiltonJacobi-Bellman partial differential equation in the Barron/Jensen-Frankowska sense. We show how this framework allows us to translate properties of capture basins into corresponding properties of the solutions to this problem. For instance, this approach provides a representation formula of the solution which boils down to the Lax-Hopf formula in the absence of constraints.
\end{abstract}

\footnotetext{
${ }^{1}$ LASTRE (Laboratoire d'Applications des Systèmes Tychastiques Régulés) 14, rue Domat, F-75005 Paris, aubin.jp@gmail.com, http://lastre.asso.fr/aubin

${ }^{2}$ Jean-Pierre Aubin thanks Giuseppe Da Prato for inviting him to the Scuola Normale di Pisa and acknowledges the financial support provided through the European Community's Human Potential Programme under contract HPRNCT-200200281 (Evolution Equations for Deterministic and Stochastic Systems).

${ }^{3}$ University of California at Berkeley, Department of Civil and Environmental Engineering. Davis Hall 711, UC Berkeley, Berkeley, CA 94720-1710. bayen@ce.berkeley.edu

${ }^{4}$ Université Paris-Dauphine, Département de Mathématiques, CRVJC, Place du Maréchal de Lattre de Tassigny, 75775 Paris Cedex 16, France
} 


\section{Introduction}

\subsection{Motivation}

This article is motivated by macroscopic fluid models of highway traffic, following the pioneering work of Lighthill, Whitham and Richards 61, 75. In their original work, the authors modelled highway traffic flow with a first order hyperbolic partial differential equation with concave flux function, called the LighthillWhitham-Richards (partial differential) equation. This model is the seminal model for numerous highway traffic flow studies available in the literature today [2, 42, 43, 60, 30, 82, 28. It models the evolution of the density of vehicles on a highway by a conservation law, in which the mathematical model of the flux function inside the conservation law results from empirical measurements $[57$.

Solutions to such equations may have shocks (they are set-valued maps), which model abrupt changes in vehicle density on the highway [2, and only model physical phenomena to a certain degree. Hence discontinuous selections of these solutions are looked after, for instance, the entropy solution [2] of Oleinik [70], which is acknowledged to be the proper weak solution of this problem. There has been an extensive literature on this problem, of which we single out the work of Bardos, Leroux and Nedelec [23, 80.

Very few results applicable to highway traffic are available for control of first order hyperbolic conservation laws. Differential flatness [4] has been successfully applied to Burgers equation (and therefore to the Lighthill-Whitham-Richards equation) in [72] order to avoid the formation of such shockwaves. This analysis does not so far extend to the presence of shocks. Lyapunov based techniques have also been applied to the Burgers equation [59]. Adjoint based methods have been successfully applied to networks of LighthillWhitham-Richards equations in [54; these results seem so far the most promising, but they do not have guarantees to provide an optimal control policy. Questions of interest in controlling first-order partial differential equations, and in particular, Lighthill-Whitham-Richards equations, are still open and difficult to solve due to the presence of shocks occurring in the solutions of these partial differential equations.

In order to alleviate the technical difficulties resulting from shocks present in solution of the LighthillWhitham-Richards equation, an alternate formulation consists in considering the cumulated number of vehicles, widely used in the transportation literature as well 67, 68, 69. The cumulative number of vehicles can be thought of as a primitive of the density over space. Formally, the evolution of the cumulated number $\mathbf{N}(t, x)$ of vehicles is the solution of an Hamilton-Jacobi (partial differential) equation of the form

$$
\frac{\partial \mathbf{N}(t, x)}{\partial t}+\psi\left(\frac{\partial \mathbf{N}(t, x)}{\partial x}\right)=\psi(v(t))
$$

where the flux function $\psi$ appearing in this Hamilton-Jacobi equation is in fact concave as shown by the empirically measured flux function of the Lighthill-Whitham-Richards equation [61, 175, 23, 80. The function $v(\cdot)$ will be regarded as a control of the Hamilton-Jacobi equation in forthcoming studies. It could for example model the inflow of vehicles at the entrance of a stretch of highway. It is a given datum in this paper.

The solution of this Hamilton-Jacobi equation has no shocks, but is not necessarily differentiable. It is only upper semicontinuous. Actually, the non differentiability of the cumulated number of vehicles is closely related to the presence of the shocks of the solution to the Lighthill-Whitham-Richards equation (see for instance [36, 37, 38]).

Since the Lighthill-Whitham-Richards equation and the Hamilton-Jacobi equation model the same physical phenomenon and since both formulations are equivalently used in the highway transportation literature, we single out in this paper the study of the evolution of the cumulated number of vehicles for benefiting of the extensive knowledge of Hamilton-Jacobi equations for which control and viability techniques can be applied. 


\subsection{Contributions of the paper}

We shall revisit this Hamilton-Jacobi equation by answering new questions:

- introducing a nontrivial right hand side,

- involve Dirichlet conditions,

- and above all, impose inequality constraints on the solution, for instance, upper bounds on the cumulated number of vehicles, depending on time and space variables.

For this purpose, we suggest to use a novel point of view based on the concept of capture basin of a target viable in an environment extensively studied in the framework of viability theory: Given a closed subset of a finite dimensional vector space regarded as an environment, a closed subset of this environment considered as a target and a control system, the viable capture basin is the subset of initial states of the environment from which starts at least one evolution governed by the control system viable in the environment until the finite time when it reaches the target (see Definition 9.3. p 25). It happens that the hypograph of the solution to the Hamilton-Jacobi equation satisfying initial and Dirichlet conditions as well as inequality constraints is the capture basin of an auxiliary target (involving initial and boundary conditions) viable in an auxiliary environment (involving inequality constraints) under an auxiliary control system (involving the flux function of the Hamilton-Jacobi equation).

Hence, anticipating on this property, we define the viability hyposolution of the Dirichlet problem for such an Hamilton-Jacobi equation with constraints from this property of being a viable capture basin (see Definition 3.1. $\mathrm{p}$ 6. Then we proceed by translating properties of viable capture basins (see [7] for instance) in the language of partial differential equations for this particular case. We shall prove that the viability hyposolution

1. is the unique generalized solution in the Barron/Jensen-Frankowska sense ${ }^{5}$ (a weaker concept of viscosity solution introduced by Crandall, Evans and Lions in [41, 40, for continuous solutions adapted to the case when solution is only semicontinuous): Theorem 8.1 p 22 .

2. is equivalently the unique upper semicontinuous solution in the contingent Frankowska sense: Theorem 7.1. $\mathrm{p} 19$,

3. satisfies the sup-linearity property and depends "hypo-continuously" of the initial and Dirichlet conditions,

4. is represented by the Lax-Hopf formula (see Theorem 4.1, p 8 ) in the absence of inequality constraints, a more involved representation formula (see Theorem 4.5. p 11) in the presence of inequality constraints, upper estimates (maximum principe, see Proposition 4.3, p.10) and lower estimates (see Proposition 4.4 $\mathrm{p} 10$.

\footnotetext{
${ }^{5}$ Hélène Frankowska proved that the epigraph of the value function of an optimal control problem - assumed to be only lower semicontinuous - is semipermeable (i.e., invariant and backward viable) under a (natural) auxiliary system. Furthermore, when it is continuous, she proved that its epigraph is viable and its hypograph invariant 50 51 53. By duality, she proved that the latter property is equivalent to the fact that the value function is a viscosity solution of the associated Hamilton-Jacobi equation in the sense of Crandall and Lions. See also 25, 21, 8, for more details. Such concepts have been extended to solutions of systems of first-order partial differential equations without boundary conditions by Hélène Frankowska and one of the authors (see $13,14,15,16,17,18$, and chapter 8 of [5]). See also 10, 11.
} 


\subsection{Outline of the paper}

In order to make the paper more readable by postponing the technical difficulties, we chose to begin by stating the problem and the main assumptions which will not be repeated all along the paper. We next define the viability hyposolution to the non homogeneous Dirichlet/initial value problem for our class of HamiltonJacobi equations under inequality constraint as the capture basin of a target summarizing the Dirichlet/initial data viable in a target associated with inequality constraints. Then, we translate the properties of capture basins to the viability hyposolution, starting with a general representation formula providing Lax-Hopf formulas in the absence of inequality constraints. We next check that the viability hyposolution satisfies the Dirichlet and initial conditions as well as the inequality constraints. The last three sections are devoted to the proof that the viability hyposolution is a solution to the Hamilton-Jacobi partial differential equation in two equivalent dual generalized sense by translating both the Viability Theorem and the Invariance Theorem characterizing the capture basin in terms of either tangential conditions or normal conditions, as it was done in a long series of papers by Hélène Frankowska. Using tangential conditions, we express the viability hyposolution as a solution to the Hamilton-Jacobi partial differential equation couched in terms of contingent hypoderivatives, whereas using normal conditions, we characterize it in terms of superdifferentials, as it was done independently by Barron/Jensen and Frankowska, in the spirit of nonsmooth analysis and viscosity solutions. The presence of inequality constraints complicates the technical formulation of the concept of solution at points where the solution touches the constraint, above all in the superdifferentials formulation, justifying the reason why we conclude this paper by this dual characterization. An appendix gathers some definitions, notations and basic prerequisites of viability theory and convex analysis for the convenience of readers who are not familiar with these topics.

\section{Statement of the Problem}

We set $X:=\mathbb{R}^{n}$. Let us consider

1. A concave function $\psi: X \mapsto \mathbb{R}$ satisfying growth conditions

$$
\forall v \in X, \beta-\sigma_{A}(v) \leq \psi(v) \leq \delta-\sigma_{A}(v)
$$

for some compact convex subset $A \subset X$, where $\sigma_{A}(v):=\sup _{u \in A}\langle u, v\rangle$ is the support function of $A$ and where $\beta \leq \delta$,

2. A bounded continuous function $v: \mathbb{R}_{+} \mapsto \operatorname{Dom}(\psi)$,

3. An upper semicontinuous initial datum $\mathbf{N}_{0}: X \mapsto \mathbb{R}_{+}$. We set $\mathbf{N}_{0}(0, x):=\mathbf{N}_{0}(x)$ and $\mathbf{N}_{0}(t, x):=-\infty$ if $t>0$.

4. A closed subset $K \subset X$ with nonempty interior $\operatorname{Int}(K)=: \Omega$ and boundary $\partial K=: \Gamma$,

5. An upper semicontinuous boundary datum $\gamma: \mathbb{R}_{+} \times X \mapsto \mathbb{R}$, satisfying ${ }^{6}$

$$
\forall x \in \partial K, \quad \mathbf{N}_{0}(x)=\gamma(0, x) \text { and } \forall t \geq 0, \quad \forall x \in \operatorname{Int}(K), \gamma(t, x)=-\infty
$$

6. A Lipschitz function $\mathbf{b}: \mathbb{R}_{+} \times X \mapsto \mathbb{R} \cup\{-\infty\}$ setting the upper constraint.

\footnotetext{
${ }^{6}$ This is not mandatory. We can take any function such that $\operatorname{Dom}(\gamma) \subset K$ is strictly contained in $K$, an instance which may be useful for defining "guards" in impulse or hybrid systems, for instance. Boundary conditions are obtained when Dom $(\gamma)=\partial K$.
} 
We shall also assume in this paper that the data satisfy the following consistency conditions

$$
\begin{cases}\text { (i) } & \forall x \in \partial K, \mathbf{N}_{0}(x)=\gamma(0, x) \\ \text { (ii) } & \forall t \geq 0, \forall x \in K, \max \left(\mathbf{N}_{0}(t, x), \gamma(t, x)\right) \leq \mathbf{b}(t, x) \\ \text { (iii) } & \forall 0 \leq r \leq s, \forall x \in \partial K, \forall y \in \partial K, \gamma(r, x)-\gamma(s, y) \leq\left\langle\frac{1}{s-r} \int_{r}^{s} v(\tau) d \tau, x-y\right\rangle \\ \text { (iv) } & \forall x \in K, \forall y \in \partial K, \quad \mathbf{N}_{0}(x) \leq \inf _{s \geq 0}\left(\gamma(s, y)+\left\langle\frac{1}{s} \int_{0}^{s} v(\tau) d \tau, x-y\right\rangle\right)\end{cases}
$$

which are needed only to prove that the Dirichlet/initial conditions are satisfied (see Theorem 5.1. p 13 below). When the function $v(\cdot) \equiv v$ is constant, they boil down to

$$
\begin{cases}(i) & \forall x \in \partial K, \mathbf{N}_{0}(x)=\gamma(0, x) \\ (\text { ii }) & \forall t \geq 0, \forall x \in K, \max \left(\mathbf{N}_{0}(t, x), \gamma(t, x)\right) \leq \mathbf{b}(t, x) \\ \text { (iii) } \quad \forall 0 \leq r \leq s, \forall x \in \partial K, y \in \partial K, \gamma(r, x)-\gamma(s, y) \leq\langle v, x-y\rangle \\ \text { (iv) } \quad \forall x \in K, y \in \partial K, \quad \mathbf{N}_{0}(x) \leq \inf _{s \geq 0} \gamma(s, y)+\langle v, x-y\rangle\end{cases}
$$

Under the above mentioned assumptions, that are assumed all along this paper, we shall solve the existence of a solution to the non-homogenous Hamilton-Jacobi equation

$$
\forall t>0, x \in \operatorname{Int}(K), \frac{\partial \mathbf{N}(t, x)}{\partial t}+\psi\left(\frac{\partial \mathbf{N}(t, x)}{\partial x}\right)=\psi(v(t))
$$

satisfying the initial and Dirichlet conditions

$$
\left\{\begin{array}{l}
\text { (i) } \quad \forall x \in K, \quad \mathbf{N}(0, x)=\mathbf{N}_{0}(x) \text { (initial condition) } \\
\text { (ii) } \quad \forall t \geq 0, \forall x \in \partial K, \mathbf{N}(t, x)=\gamma(t, x) \text { (Dirichlet boundary condition) }
\end{array}\right.
$$

and the viability constraints

$$
\forall t \geq 0, x \in K, \mathbf{N}(t, x) \leq \mathbf{b}(t, x) \text { (upper inequality constraint) }
$$

Example: - This equation is motivated by a commonly used first order model equation in highway traffic (Lighthill-Whitham-Richards equation) when $X:=\mathbb{R}$ and $K:=[\xi,+\infty[, \psi$ a concave flux function vanishing at density 0 and at a jam density $\omega>0$ and $\mathbf{N}(t, x)$ is the cumulated number of vehicles at time $t$ and at location $x \in K$. Consistency conditions (1), p 5 read in this case: $\mathbf{N}_{0}(\xi)=\gamma(0, \xi)$ and

$$
\begin{cases}\text { (i) } & \forall t \geq 0, \forall x \in K, \max \left(\mathbf{N}_{0}(t, x), \gamma(t, x)\right) \leq \mathbf{b}(t, x) \\ \text { (ii) } \quad \forall 0 \leq r \leq s, \gamma(r, \xi)-\gamma(s, \xi) \leq 0 \text { (monotonocity) } \\ \text { (iii) } \quad \forall x \in K, \mathbf{N}_{0}(x) \leq \inf _{s \geq 0}\left(\gamma(s, \xi)+\left\langle\frac{1}{s} \int_{0}^{s} v(\tau) d \tau, x-\xi\right\rangle\right)\end{cases}
$$

Then the trapezoidal flux function (such as the one proposed by Daganzo [42, 43]) defined by

$$
\psi(v)= \begin{cases}\nu^{b} v & \text { if } v \leq \gamma^{b} \\ \delta & \text { if } v \in\left[\gamma^{b}, \gamma^{\sharp}\right] \\ \nu^{\sharp}(\omega-v) & \text { if } v \geq \gamma^{\sharp}\end{cases}
$$

and the Greenshield flux function

$$
\psi(v)= \begin{cases}\nu v & \text { if } \quad v \leq 0 \\ \frac{\nu}{\omega} v(\omega-v) & \text { if } \quad v \in[0, \omega] \\ \nu(\omega-v) & \text { if } \quad v \geq \omega\end{cases}
$$


satisfy the assumptions on the function $\psi$ with $A:=\left[-\nu^{b},+\nu^{\sharp}\right]$ and $A:=[-\nu,+\nu]$ respectively (see Lemma $6.2 \mathrm{p} 16$.

We characterize the solution to this non-homogenous Dirichlet/Initial Value problem with inequality constraints through the capture basin of a target defined by the Dirichlet/initial conditions viable in an environment defined by inequality constraints under an adequate control system.

\section{The Viability Hyposolution}

The assumption that the flux function $\psi$ is concave and upper semicontinuous plays a crucial role for defining the viability hyposolution. Indeed, the Fenchel theorem allows us to characterize it by

$$
\psi(p)=\inf _{u \in \operatorname{Dom}\left(\varphi^{\star}\right)}\left[\varphi^{\star}(u)-\langle p, u\rangle\right]
$$

where $\varphi^{\star}$ is the Fenchel conjugate function, which is the convex lower semicontinuous function defined by

$$
\varphi^{\star}(u):=\sup _{p \in \operatorname{Dom}(\psi)}[\langle p, u\rangle+\psi(p)]
$$

We introduce the auxiliary characteristic control system:

$$
\left\{\begin{array}{l}
\tau^{\prime}(t)=-1 \\
x^{\prime}(t)=u(t) \\
y^{\prime}(t)=\varphi^{\star}(u(t))-\psi(v(\tau(t))) \text { where } u(t) \in \operatorname{Dom}\left(\varphi^{\star}\right)
\end{array}\right.
$$

To be rigorous, we have to mention once and for all that the controls $u(\cdot)$ are measurable integrable functions with values in $\operatorname{Dom}\left(\varphi^{\star}\right)$, and thus, ranging $L^{1}\left(0, \infty ; \operatorname{Dom}\left(\varphi^{\star}\right)\right)$, and that the above system of differential equations is valid for almost all $t \geq 0$.

We set $\mathbf{c}(t, x):=\max \left(\mathbf{N}_{0}(t, x), \gamma(t, x)\right)$, defined by

$$
\mathbf{c}(t, x):= \begin{cases}-\infty & \text { if } \quad t>0 \text { and } x \in \Omega:=\operatorname{Int}(K) \\ \mathbf{N}_{0}(x) & \text { if } \quad t=0 \text { and } x \in K \\ \gamma(t, x) & \text { if } \quad t \geq 0 \text { and } x \in \Gamma:=\partial K\end{cases}
$$

We introduce the environment $\mathcal{K}:=\mathcal{H} y p(\mathbf{b})$ is the subset of triples $(T, x, y) \subset \mathbb{R}_{+} \times X \times \mathbb{R}$ such that $y \leq \mathbf{b}(T, x)$ (this is the hypograph of the function $\mathbf{b}$ ) and the target $\mathcal{C}:=\mathcal{H} y p(\mathbf{c})$ the subset of triples $(T, x, y) \subset \mathbb{R}_{+} \times X \times \mathbb{R}$ such that $y \leq \mathbf{c}(T, x)$ (which is the hypograph of the function $\mathbf{c}$ ).

Definition 3.1 The Viability Hyposolution. The capture basin $\operatorname{Capt}_{87}(\mathcal{K}, \mathcal{C})$ of a target $\mathcal{C}$ viable in the environment $\mathcal{K}$ under control system (8) is the subset of initial states $(t, x, y)$ such that there exists a measurable control $u(\cdot)$ such that the associated solution

$$
s \mapsto\left(t-s, x+\int_{0}^{s} u(\tau) d \tau, y+\int_{0}^{s}\left(\varphi^{\star}(u(\tau))-\psi(v(t-\tau))\right) d \tau\right)
$$

is viable in $\mathcal{K}$ until it reaches the target $\mathcal{C}$.

The viability hyposolution $\mathbf{N}$ is defined by

$$
\mathbf{N}(t, x):=\sup _{(t, x, y) \in \operatorname{Capt}_{[}} y
$$


We shall prove the following:

Theorem 3.2 Non-homogenous Dirichlet/Initialvalue Problem with inequality contraints. The viability hyposolution $\mathbf{N}$ defined by (9) is the largest upper semicontinuous solution to Hamilton-Jacobi equation (2) satisfying initial and Dirichlet conditions (3) and inequality constraints (4) in both the contingent solution sense (see (22), $p$ (19) and in the contingent normal sense (see (25), $p$ 22). If the functions $\psi, \varphi^{\star}$ and $v$ are furthermore Lipschitz, then the viability hyposolution $\mathbf{N}$ is its unique upper semicontinuous solution in both the contingent Frankowska sense (see (23) and (24), $p$ 19) and in the Barron-Jensen/Frankowska sense (see 28) and 27), $p$ 20 and Theorems 7.1. $p$ 19 and 8.1. $p$ 20. for the precise statement).

We shall derive this theorem and other results from the properties of capture basins gathered in [7, 9].

Since the capture basin of a union of targets is the union of the capture basins of these targets, we infer that whenever $\mathbf{c}:=\sup _{i} \mathbf{c}_{i}$ is the upper enveloppe of a family of functions $\mathbf{c}_{i}$, then the viability hyposolution is the upper enveloppe

$$
\forall t \geq 0, x \in X, \mathbf{N}(t, x)=\sup _{i} \mathbf{N}_{\mathbf{c}_{i}}(t, x)
$$

of the solutions $\mathbf{N}_{\mathbf{c}_{i}}$ (sup-linearity property).

In particular, since $\mathbf{c}(t, x):=\max \left(\mathbf{N}_{0}(t, x), \gamma(t, x)\right.$ ) (extended to $-\infty$ when $t>0$ or $x \in \operatorname{Int}(K)$ ), we obtain the decomposition formula

$$
\mathbf{N}(t, x)=\max \left(\mathbf{N}_{N_{0}}(t, x), \mathbf{N}_{\gamma}(t, x)\right)
$$

in terms of initial condition component $\mathbf{N}_{\mathbf{N}_{0}}$ and the Dirichlet component $\mathbf{N}_{\gamma}$ of the viability hyposolution $\mathbf{N}$ defined by

$$
\left\{\begin{array}{l}
\mathbf{N}_{\mathbf{N}_{0}}(t, x):=\sup _{(t, x, y) \in \operatorname{Capt}_{\boldsymbol{\nabla}}\left(\mathcal{H} y p(\mathbf{b}), \mathcal{H} y p\left(\mathbf{N}_{0}\right)\right)} y \\
\mathbf{N}_{\gamma}(t, x):=\sup _{(t, x, y) \in \operatorname{Capt}_{\mathbf{8}}}(\mathcal{H} y p(\mathbf{b}), \mathcal{H} y p(\gamma))
\end{array}\right.
$$

The viability hyposolution depends continuously on the data in the following sense: If the hypographs of a sequence of initial data $\mathbf{c}_{j}$ converge in the upper Painlevé-Kuratowski sense (see for instance [12]) to the hypographs of data $\mathbf{c}$, then the upper Painlevé-Kuratowski limit of the hypographs of the solutions $\mathbf{N}_{j}$ associated with data $\mathbf{c}_{j}$ is contained in the hypograph of the hyposolution $\mathbf{N}$ associated with data $\mathbf{c}$ (upper hypocontinuity property). If the functions $\psi, \varphi^{\star}$ and $v$ are furthermore Lipschitz, the hypograph of the hyposolution $\mathbf{N}$ associated with data $\mathbf{c}$ is contained in the lower Painlevé-Kuratowski limit of the hypographs of the solutions $\mathbf{N}_{j}$ associated with data $\mathbf{c}_{j}$ (lower hypocontinuity property), so that both the upper and lower limits coincide with the hypograph of the hyposolution $\mathbf{N}$ (hypoconvergence of the solutions, defined in [12] or [76] for instance). These statements follow from Theorem 6.6 of [7 stating that if the system is both Marchaud and Lipschitz, the capture basin of a Painlevé-Kuratowski limit of targets is the Painlevé-Kuratowski limit of the capture basins of the targets.

\section{The Lax-Hopf formula}

When there is no inequality constraint, we prove that the viability hyposolution can be represented explicitly as a simple maximization problem involving the Fenchel conjugate $\varphi^{\star}$ defined by (7). 
Theorem 4.1 The Lax-Hopf Formula. Let us consider the case without inequality constraints and set

$$
\tau(x, u):=\inf _{x+t u \notin K} t \quad \text { and } \quad \sigma(t, x, u):=\min (t, \tau(x, u))
$$

Then the viability hyposolution [12], $p 8$ can be written

$$
\left\{\begin{array}{l}
\mathbf{N}(t, x) \\
=\sup _{\left\{u \in \operatorname{Dom}\left(\varphi^{\star}\right)\right\}}\left(\mathbf{c}(t-\sigma(t, x, u), x+\sigma(t, x, u) u)-\sigma(t, x, u) \varphi^{\star}(u)\right)+\int_{t-\sigma(t, x, u)}^{t}(\psi(v(\tau))) d \tau
\end{array}\right.
$$

Using the decomposition $\mathbf{N}(t, x)=\max \left(\mathbf{N}_{N_{0}}(t, x), \mathbf{N}_{\gamma}(t, x)\right)$, we derive the more explicit formula

$$
\left\{\begin{array}{l}
\mathbf{N}_{N_{0}}(t, x)=\sup _{u \in \operatorname{Dom}\left(\varphi^{\star}\right)}\left(\mathbf{N}_{0}(x+t u)-t \varphi^{\star}(u)\right)+\int_{0}^{t} \psi(v(\tau)) d \tau \\
\mathbf{N}_{\gamma}(t, x)=\sup _{\left\{u \in \operatorname{Dom}\left(\varphi^{\star}\right) \mid \tau(x, u) \leq t\right\}}\left[\gamma(t-\tau(x, u), x+\tau(x, u) u)-\tau(x, u) \varphi^{\star}(u)\right] \\
+\int_{t-\tau(x, u)}^{t} \psi(v(\tau)) d \tau
\end{array}\right.
$$

involving the initial and Dirichlet condition.

Proof - Let us associate with $u(\cdot)$

$$
\tau(x, u(\cdot)):=\inf _{x+\int_{0}^{t} u(\tau) d \tau \notin K} t \quad \text { and } \quad \sigma(t, x, u(\cdot))=\min (t, \tau(x, u(\cdot)))
$$

The formula is derived from the general representation formula

$$
\left\{\begin{array}{l}
\mathbf{N}(t, x)=\sup _{u(\cdot)}\left(\mathbf{c}\left(t-\sigma(t, x, u(\cdot)), x+\int_{0}^{\sigma(t, x, u(\cdot))} u(\tau) d \tau\right)-\int_{0}^{\sigma(t, x, u(\cdot))} \varphi^{\star}(u(\tau)) d \tau\right) \\
+\int_{t-\sigma(t, x, u(\cdot))}^{t} \psi(v(\tau)) d \tau
\end{array}\right.
$$

of the viability hyposolution without constraints given by Corollary 4.6, p. 13

1. Taking constant controls $u(\cdot) \equiv u$ and observing that $\tau(x, u)=\tau(x, u(\cdot))$, we infer that

$$
\sup _{u \in \operatorname{Dom}\left(\varphi^{\star}\right)}\left(\mathbf{c}(t-\sigma(t, x, u), x+\sigma(t, x, u) u)-\sigma(t, x, u) \varphi^{\star}(u)\right)+\int_{t-\sigma(t, x, u)}^{t} \psi(v(\tau)) d \tau \leq \mathbf{N}(t, x)
$$

2. Let us associate with $u(\cdot)$ the function $\widehat{u}$ defined by $\widehat{u}(s):=\frac{1}{s} \int_{0}^{s} u(\tau) d \tau$. We first observe that

$$
\tau(x, u(\cdot))=\tau(x, \widehat{u}(\tau(x, u(\cdot))))
$$

Since $\varphi^{\star}$ is convex and lower semicontinuous and $\psi$ is concave and upper semicontinuous, Jensen inequality implies 


$$
\varphi^{\star}\left(\frac{1}{s} \int_{0}^{s} u(\tau) d \tau\right) \leq \frac{1}{s} \int_{0}^{s} \varphi^{\star}(u(\tau)) d \tau \text { and } \frac{1}{s} \int_{0}^{s} \psi(v(t-\tau)) d \tau \leq \psi\left(\frac{1}{s} \int_{t-s}^{t} v(\tau) d \tau\right)
$$

and thus

$$
\int_{0}^{s} \psi(v(t-\tau)) d \tau-\int_{0}^{s} \varphi^{\star}(u(\tau)) d \tau \leq s\left(\psi\left(\frac{1}{s} \int_{t-s}^{t} v(\tau) d \tau\right)-\varphi^{\star}(\widehat{u}(s))\right)
$$

Consequently, setting $t^{\sharp}:=\sigma(t, x, u(\cdot))=\tau(x, \widehat{u}(\sigma(t, x, u(\cdot))))$ and $u^{\sharp}:=\widehat{u}\left(t^{\sharp}\right)$, we obtain inequalities

$$
\left\{\begin{array}{l}
\mathbf{c}\left(t-t^{\sharp}, x+\int_{0}^{t^{\sharp}} u(\tau) d \tau\right)-\int_{0}^{t^{\sharp}} \varphi^{\star}(u(\tau)) d \tau+\int_{t-t^{\sharp}}^{t} \psi(v(\tau)) d \tau \\
\leq \mathbf{c}\left(t-t^{\sharp}, x+t^{\sharp} u^{\sharp}\right)-t^{\sharp} \varphi^{\star}\left(u^{\sharp}\right)+\int_{t-t^{\sharp}}^{t} \psi(v(\tau)) d \tau \\
\leq \sup _{\left\{u \in \operatorname{Dom}\left(\varphi^{\star}\right)\right\}}\left(\mathbf{c}(t-\sigma(t, x, u), x+\sigma(t, x, u) u)-\sigma(t, x, u) \varphi^{\star}(u)\right)+\int_{t-\sigma(t, x, u)}^{t} \psi(v(\tau)) d \tau
\end{array}\right.
$$

Therefore, by taking the supremum, we obtain

$$
\mathbf{N}(t, x) \leq \sup _{u \in \operatorname{Dom}\left(\varphi^{\star}\right)}\left(\mathbf{c}(t-\sigma(t, x, u), x+\sigma(t, x, u) u)-\sigma(t, x, u) \varphi^{\star}(u)\right)+\int_{t-\sigma(t, x, u)}^{t} \psi(v(\tau)) d \tau
$$

This completes the proof of the Lax-Hopf inequality.

Corollary 4.2 Case of the Traffic Model. When $X:=\mathbb{R}, K:=[\xi,+\infty[, \psi$ is a concave flux function vanishing at density 0 and at a jam density $\omega>0$ and $\mathbf{N}(t, x)$ is the cumulated number of vehicles at time $t$ and at location $x \in K$, consistency conditions (5), $p$ 酒ply the existence of a unique upper semicontinuous solution $\mathbf{N}(t, x)=\max \left(\mathbf{N}_{N_{0}}(t, x), \mathbf{N}_{\gamma}(t, x)\right)$ to this problem in the Barron-Jensen/Frankowska sense satisfying the Lax-Hopf formula:

$$
\left\{\begin{array}{l}
\mathbf{N}_{N_{0}}(t, x)=\sup _{u \in \operatorname{Dom}\left(\varphi^{\star}\right)}\left(\mathbf{N}_{0}(x+t u)-t \varphi^{\star}(u)+\int_{0}^{t} \psi(v(t-\tau)) d \tau\right) \\
\mathbf{N}_{\gamma}(t, x)=\sup _{\left\{u \in \operatorname{Dom}\left(\varphi^{\star}\right) \mid u \leq \frac{\xi-x}{t}\right\}}\left(\gamma\left(t-\frac{\xi-x}{u}, \xi\right)-\frac{\xi-x}{u} \varphi^{\star}(u)+\int_{0}^{\frac{\xi-x}{u}} \psi(v(t-\tau)) d \tau\right)
\end{array}\right.
$$

We provide an upper estimate: 
Proposition 4.3 Upper Estimate of the Viability Hyposolution The viability hyposolution satisfies

$$
\mathbf{N}(t, x) \leq \sup _{u \in \operatorname{Dom}\left(\varphi^{\star}\right)}\left(\mathbf{c}(t-\sigma(t, x, u), x+\sigma(t, x, u) u)-\left\langle u, \int_{t-\sigma(t, x, u)}^{t} v(\tau) d \tau\right\rangle\right)
$$

Consequently, the viability hyposolution satisfies the following (a posteriori instead of a priori) estimate

$$
\mathbf{N}(t, x) \leq \sup _{t \geq 0, x \in K} \mathbf{c}(t, x)+t \operatorname{Diam}\left(\operatorname{Dom}\left(\varphi^{\star}\right)\right) \sup _{t \geq 0}\|v(t)\|
$$

(maximum principle)

Proof - Fix $u \in \operatorname{Dom}\left(\varphi^{\star}\right)$ and set $\sigma(t, x, u)=: s$. Definition (7), p 6 of the conjugate function implies

$$
\psi\left(\frac{1}{s} \int_{t-s}^{t} v(\tau) d \tau\right)-\varphi^{\star}(u) \leq-\left\langle\frac{1}{s} \int_{t-s}^{t} v(\tau) d \tau, u\right\rangle
$$

Consequently

$$
\left\{\begin{array}{l}
\mathbf{c}(t-\sigma(t, x, u), x+\sigma(t, x, u) u)-\sigma(t, x, u) \varphi^{\star}(u)+\int_{t-\sigma(t, x, u)}^{t}(\psi(v(\tau))) d \tau \\
\leq \mathbf{c}(t-\sigma(t, x, u), x+\sigma(t, x, u) u)-\left\langle u, \int_{t-\sigma(t, x, u)}^{t} v(\tau) d \tau\right\rangle \\
\leq \sup _{w \in \operatorname{Dom}\left(\varphi^{\star}\right)}\left(\mathbf{c}(t-\sigma(t, x, w), x+\sigma(t, x, w) w)-\left\langle w, \int_{t-\sigma(t, x, w)}^{t} v(\tau) d \tau\right\rangle\right)
\end{array}\right.
$$

Taking the supremum over $u \in \operatorname{Dom}\left(\varphi^{\star}\right)$, Lax-Hopf formula 111, $\mathrm{p} 8$ implies the upper estimate

$$
\mathbf{N}(t, x) \leq \sup _{u \in \operatorname{Dom}\left(\varphi^{\star}\right)}\left(\mathbf{c}(t-\sigma(t, x, u), x+\sigma(t, x, u) u)-\left\langle u, \int_{t-\sigma(t, x, u)}^{t} v(\tau) d \tau\right\rangle\right)
$$

This completes the proof.

Proposition 4.4 Lower Estimate. Assume that $v(t):=v$ is constant and, for simplicity, that the function $\psi$ is differentiable. Then

$$
\mathbf{c}\left(t-\sigma\left(t, x,-\psi^{\prime}(v)\right), x-\sigma\left(t, x,-\psi^{\prime}(v)\right) \psi^{\prime}(v)\right)+\sigma\left(t, x, \psi^{\prime}(v)\right)\left\langle v, \psi^{\prime}(v)\right\rangle \leq \mathbf{N}(t, x)
$$

Consequently, the hyposolution is nonnegative on its positivity domain $\operatorname{Dom}_{+}(\mathbf{N})$, defined as the subset of pairs $(t, x) \in \mathbb{R}_{+} \times K$ such that

$$
\mathbf{c}\left(t-\sigma\left(t, x,-\psi^{\prime}(v)\right), x-\sigma\left(t, x,-\psi^{\prime}(v)\right) \psi^{\prime}(v)\right)+\sigma\left(t, x,-\psi^{\prime}(v)\right)\left\langle v, \psi^{\prime}(v)\right\rangle \geq 0
$$

Proof - By definition 9.9 p 28 of the superdifferential,

$$
\forall u \in \partial_{+} \psi(v), \quad \psi(v)-\varphi^{\star}(-u)=\langle v, u\rangle
$$

Therefore, if $\psi$ is differentiable, taking $u:=-\psi^{\prime}(v)$ the unique element of $-\partial_{+} \psi(v)=\partial_{-} \varphi(v)$, Legendre equality $\psi(v)-\varphi^{\star}\left(-\psi^{\prime}(v)\right)=\left\langle v, \psi^{\prime}(v)\right\rangle$ yields 


$$
\left\{\begin{array}{l}
\mathbf{c}\left(t-\sigma\left(t, x,-\psi^{\prime}(v)\right), x-\sigma\left(t, x,-\psi^{\prime}(v)\right) \psi^{\prime}(v)\right)+\sigma\left(t, x,-\psi^{\prime}(v)\right)\left\langle v, \psi^{\prime}(v)\right\rangle \\
=\mathbf{c}(t-\sigma(t, x, u), x+\sigma(t, x, u) u)-\sigma(t, x, u)\langle v, u\rangle \\
=\mathbf{c}(t-\sigma(t, x, u), x+\sigma(t, x, u) u)+\sigma(t, x, u)\left(\psi(v)-\varphi^{\star}(u)\right) \leq \mathbf{N}(t, x)
\end{array}\right.
$$

thanks to the Lax-Hopf formula.

Theorem 4.5 Representation Formula of the viability solution (case with constraints). We already set

$$
\tau(x, u(\cdot)):=\inf _{x+\int_{0}^{t} u(\tau) d \tau \notin K} t \text { and } \sigma(t, x, u(\cdot))=\min (t, \tau(x, u(\cdot)))
$$

The viability hyposolution can be represented in the form:

$$
\left\{\begin{array}{l}
\mathbf{N}(t, x)=\sup _{u(\cdot)}[\max ( \\
\mathbf{c}\left(t-\sigma(t, x, u(\cdot)), x+\int_{0}^{\sigma(t, x, u(\cdot))} u(\tau) d \tau\right)-\int_{0}^{\sigma(t, x, u(\cdot))} \varphi^{\star}(u(\tau)) d \tau+\int_{t-\sigma(t, x, u(\cdot))}^{t} \psi(v(\tau)) d \tau, \\
\left.\left.\left.\inf _{s \in[0, \sigma(t, x, u(\cdot))]}\left(\mathbf{b}\left(t-s, x+\int_{0}^{s}\right) u(\tau) d \tau\right)-\int_{0}^{s} \varphi^{\star}(u(\tau)) d \tau+\int_{t-s}^{t} \psi(v(\tau)) d \tau\right)\right)\right]
\end{array}\right.
$$

Using the decomposition $\mathbf{N}(t, x)=\max \left(\mathbf{N}_{N_{0}}(t, x), \mathbf{N}_{\gamma}(t, x)\right)$, this formula boils down to

$$
\left\{\begin{array}{l}
\mathbf{N}_{N_{0}}(t, x)=\sup _{u(\cdot)}\left[\operatorname { m a x } \left(\mathbf{N}_{0}\left(x+\int_{0}^{t} u(\tau) d \tau\right)-\int_{0}^{t} \varphi^{\star}(u(\tau)) d \tau+\int_{0}^{t} \psi(v(\tau)) d \tau,\right.\right. \\
\left.\inf _{s \in[0, t]}\left(\mathbf{b}\left(t-s, x+\int_{0}^{s} u(\tau) d \tau\right)-\int_{0}^{s} \varphi^{\star}(u(\tau)) d \tau+\int_{t-s}^{t} \psi(v(\tau)) d \tau\right)\right]
\end{array}\right.
$$

and

$$
\left\{\begin{array}{l}
\mathbf{N}_{\gamma}(t, x)=\sup _{\{u(\cdot) \mid \tau(x, u(\cdot)) \leq t\}}[\max ( \\
\gamma\left(t-\tau(x, u(\cdot)), x+\int_{0}^{\tau(x, u(\cdot))} u(\tau) d \tau\right)-\int_{0}^{\tau(x, u(\cdot))} \varphi^{\star}(u(\tau)) d \tau+\int_{t-\tau(x, u(\cdot))}^{t} \psi(v(\tau)) d \tau, \\
\left.\left.\inf _{s \in[0, \tau(x, u(\cdot))]}\left(\mathbf{b}\left(t-s, x+\int_{0}^{s} u(\tau) d \tau\right)-\int_{0}^{s} \varphi^{\star}(u(\tau)) d \tau+\int_{t-s}^{t} \psi(v(\tau)) d \tau\right)\right)\right]
\end{array}\right.
$$

Proof - We begin by observing that a solution $(\tau(\cdot), x(\cdot), y(\cdot))$ to control system $(8)$ starting from $(t, x, y)$ is given by $\tau(s)=t-s, x(s)=x+\int_{0}^{s} u(r) d r$ and

$$
y(s)=y+\int_{0}^{s}\left(\varphi^{\star}(u(r))-\psi(v(t-r))\right) d r
$$

for some $u(\cdot)$.

Therefore, to say that $(t, x, y)$ belongs to the capture basin $\operatorname{Capt}_{8}(\mathcal{K}, \mathcal{C})$ amounts to saying that there exist a solution $(\tau(\cdot), x(\cdot), y(\cdot))$ to the characteristic control system [8] starting from $(t, x, y)$ and $t^{\star} \in[0, t]$ such that 
1. $\left(t-t^{\star}, x\left(t^{\star}\right), y\left(t^{\star}\right)\right)$ belongs to the target $\mathcal{C}$, i.e., such that

$$
y\left(t^{\star}\right):=y+\int_{0}^{t^{\star}}\left(\varphi^{\star}(u(\tau))-\psi(v(t-\tau))\right) d \tau \leq \mathbf{c}\left(t-t^{\star}, x\left(t^{\star}\right)\right)=\mathbf{c}\left(t-t^{\star}, x+\int_{0}^{t^{\star}} u(\tau) d \tau\right)
$$

2. $\forall s \in\left[0, t^{\star}\right],(t-s, x(s), y(s))$ belongs to the environment $\mathcal{K}$, i.e., such that

$$
y(s)=y+\int_{0}^{s}\left(\varphi^{\star}(u(\tau))-\psi(v(t-\tau))\right) d \tau \leq \mathbf{b}(t-s, x(s))=\mathbf{b}\left(t-s, x+\int_{0}^{s} u(\tau) d \tau\right)
$$

This implies that

$$
y \leq \min \left(\begin{array}{c}
\mathbf{c}\left(t-t^{\star}, x+\int_{0}^{t^{\star}} u(\tau) d \tau\right)-\int_{0}^{t^{\star}}\left(\varphi^{\star}(u(\tau))-\psi(v(t-\tau))\right) d \tau \\
\inf _{s \in\left[0, t^{\star}\right]} \mathbf{b}\left(t-s, x+\int_{0}^{s} u(\tau) d \tau\right)-\int_{0}^{s}\left(\varphi^{\star}(u(\tau))-\psi(v(t-\tau))\right) d \tau
\end{array}\right)
$$

Since $y$ is finite, this implies that $\mathbf{c}\left(t-t^{\star}, x+\int_{0}^{t^{\star}} u(\tau) d \tau\right)$ must be finite, and thus, that

1. either $t-t^{\star}=0$, in which case $\mathbf{c}\left(t-t^{\star}, x+\int_{0}^{t^{\star}} u(\tau) d \tau\right)=\mathbf{N}_{0}\left(x+\int_{0}^{t} u(\tau) d \tau\right)$

2. or $x\left(t^{\star}\right) \in \partial K$, which means that $t^{\star}=\tau(x, u(\cdot))=\sigma(t, x, u(\cdot)) \leq t$, in which case $\mathbf{c}\left(t-t^{\star}, x+\int_{0}^{t^{\star}} u(\tau) d \tau\right)=\gamma\left(t-\sigma(t, x, u(\cdot)), x+\int_{0}^{\sigma(t, x, u(\cdot))} u(\tau) d \tau\right)$.

This implies that $\mathbf{N}(t, x) \leq \mathbf{V}(t, x)$ where

$$
\left\{\begin{array}{l}
\mathbf{V}(t, x)=\sup _{u(\cdot)}(\max ( \\
\mathbf{c}\left(t-\sigma(t, x, u(\cdot)), x+\int_{0}^{\sigma(t, x, u(\cdot))} u(\tau) d \tau\right)-\int_{0}^{\sigma(t, x, u(\cdot))} \varphi^{\star}(u(\tau)) d \tau+\int_{t-\sigma(t, x, u(\cdot))}^{t} \psi(v(\tau)) d \tau, \\
\left.\inf _{s \in[0, \sigma(t, x, u(\cdot))]}\left(\mathbf{b}\left(t-s, x+\int_{0}^{s} u(\tau) d \tau\right)-\int_{0}^{s} \varphi^{\star}(u(\tau)) d \tau+\int_{t-s}^{t} \psi(v(\tau)) d \tau\right)\right)
\end{array}\right.
$$

For proving the converse inequality, we associate with every $\varepsilon>0$ a control $t \mapsto u_{\varepsilon}(t) \in \operatorname{Dom}\left(\varphi^{\star}\right)$ such that

$$
\left\{\begin{array}{l}
\mathbf{V}(t, x)-\varepsilon \leq \max ( \\
\mathbf{c}\left(t-\sigma\left(t, x, u_{\varepsilon}(\cdot)\right), x+\int_{0}^{\sigma\left(t, x, u_{\varepsilon}(\cdot)\right)} u_{\varepsilon}(\tau) d \tau\right)-\int_{0}^{\sigma\left(t, x, u_{\varepsilon}(\cdot)\right)} \varphi^{\star}\left(u_{\varepsilon}(\tau)\right) d \tau+\int_{t-\sigma\left(t, x, u_{\varepsilon}(\cdot)\right)}^{t} \psi(v(\tau)) d \tau, \\
\left.\inf _{s \in\left[0, \sigma\left(t, x, u_{\varepsilon}(\cdot)\right)\right]}\left(\mathbf{b}\left(t-s, x+\int_{0}^{s} u(\tau) d \tau\right)-\int_{0}^{s} \varphi^{\star}\left(u_{\varepsilon}(\tau)\right) d \tau+\int_{t-s}^{t} \psi(v(\tau)) d \tau\right)\right)
\end{array}\right.
$$

Therefore, setting $x_{\varepsilon}(t):=x+\int_{0}^{t} u_{\varepsilon}(s) d s$ and 


$$
y_{\varepsilon}(t):=\mathbf{V}(t, x)-\varepsilon+\int_{0}^{t}\left(\varphi^{\star}\left(u_{\varepsilon}(r)\right)-\psi(v(t-r))\right) d r
$$

we observe that the function $s \mapsto\left(t-s, x_{\varepsilon}(s), y_{\varepsilon}(s)\right)$ starts from $(t, x, \mathbf{V}(t, x)-\varepsilon)$, is a solution to characteristic control system (8), viable in $\mathcal{K}$ for $s \leq \sigma\left(t, x, u_{\varepsilon}(\cdot)\right)$ because

$$
y_{\varepsilon}(s)=\mathbf{V}(t, x)-\varepsilon+\int_{0}^{s}\left(\varphi^{\star}\left(u_{\varepsilon}(r)\right)-\psi(v(t-r)) d r\right) \leq \mathbf{b}\left(t-s, x_{\varepsilon}(s)\right)
$$

and reaching the target $\mathcal{C}:=\mathcal{H} y p(\mathbf{c})$ at time $t_{\varepsilon}:=\sigma\left(t, x, u_{\varepsilon}(\cdot)\right)$,

$$
y_{\varepsilon}\left(t_{\varepsilon}\right)=\mathbf{V}(t, x)-\varepsilon+\int_{0}^{t_{\varepsilon}}\left(\varphi^{\star}\left(u_{\varepsilon}(r)\right)-\psi(v(t-r))\right) d r \leq \mathbf{c}\left(t-t_{\varepsilon}, x_{\varepsilon}\left(t_{\varepsilon}\right)\right)
$$

This implies that $(t, x, \mathbf{V}(t, x)-\varepsilon)$ belongs to the capture basin Capt ${ }_{88}(\mathcal{K}, \mathcal{C})$, and thus, that $\mathbf{V}(t, x)-\varepsilon \leq$ $\mathbf{N}(t, x)$. Letting $\varepsilon$ converge to 0 provides the converse inequality, and thus, the representation formula we were looking for.

Corollary 4.6 Representation Formula of the viability solution (case without constraints). Without inequality constraints, the viability hyposolution can be represented in the form:

$$
\left\{\begin{array}{l}
\mathbf{N}(t, x)=\sup _{u(\cdot)}\left(\int_{t-\sigma(t, x, u(\cdot))}^{t} \psi(v(\tau)) d \tau\right. \\
\left.+\mathbf{c}\left(t-\sigma(t, x, u(\cdot)), x+\int_{0}^{\sigma(t, x, u(\cdot))} u(\tau) d \tau\right)-\int_{0}^{\sigma(t, x, u(\cdot))} \varphi^{\star}(u(\tau)) d \tau\right)
\end{array}\right.
$$

\section{Dirichlet/Initial Conditions and Inequality Constraints}

We begin by checking that the viability hyposolution satisfies the initial condition, the Dirichlet condition and the inequality constraints:

Theorem 5.1 Dirichlet/Initial Conditions and Inequality Constraints. Consistency conditions (1), $p$.5 imply that the viability hyposolution satisfies the initial and Dirichlet conditions (3), $p .5$ and inequality contraints (4), $p$.5.

Proof - Inclusions

$$
\mathcal{C}:=\mathcal{H}_{y p}(\mathbf{c}) \subset \operatorname{Capt}_{\mathbb{8}}(\mathcal{K}, \mathcal{C}) \subset \mathcal{K}:=\mathcal{H} y p(\mathbf{b})
$$

imply that

$$
\forall t \geq 0, \forall x \in K, \mathbf{c}(t, x) \leq \mathbf{N}(t, x) \leq \mathbf{b}(t, x)
$$

and thus inequality constraint $\mathbf{N}(t, x) \leq \mathbf{b}(t, x)$ and inequalities $\mathbf{N}_{0}(x) \leq \mathbf{N}(0, x)$ for all $x \in K$ and $\gamma(t, x) \leq$ $\mathbf{N}(t, x)$ for all $t \geq 0$ and $x \in \partial K$. We now prove by contradiction that consistency conditions (1), $\mathrm{p} .5$ imply 
converse inequalities $\mathbf{N}_{0}(x) \geq \mathbf{N}(0, x)$ for all $x \in K$ and $\gamma(t, x) \geq \mathbf{N}(t, x)$ for all $t \geq 0$ and $x \in \partial K$ that we summarize in

$$
\forall(t, x) \in \operatorname{Dom}(\mathbf{c}), \quad \mathbf{N}(t, x) \leq \mathbf{c}(t, x)
$$

Assume that there exist $(t, \xi) \in \operatorname{Dom}(\mathbf{c})$ and $\varepsilon>0$ such that

$$
\mathbf{N}(t, \xi)=\mathbf{c}(t, \xi)+\varepsilon
$$

Since $(t, \xi, \mathbf{N}(t, \xi))$ belongs to the capture basin $\operatorname{Capt}_{\sqrt{8}}(\mathcal{H} y p(\mathbf{b}), \mathcal{H} y p(\mathbf{c}))$, there exist a solution $(\tau(\cdot), x(\cdot), y(\cdot))$ to the characteristic control system (8) starting from $(t, \xi, \mathbf{N}(t, \xi))$ and $t^{\star}>0$ such that $\left(t-t^{\star}, x\left(t^{\star}\right), y\left(t^{\star}\right)\right)$ belongs to the hypograph $\mathcal{H} y p(\mathbf{c})$ : Setting $x\left(t^{\star}\right)=\xi+\int_{0}^{t^{\star}} u(\tau) d \tau=\eta$, we obtain

$$
y\left(t^{\star}\right)=\mathbf{N}(t, \xi)+\int_{0}^{t^{\star}} \varphi^{\star}(u(\tau)) d \tau-\int_{0}^{t^{\star}} \psi(v(t-\tau)) d \tau \leq \mathbf{c}\left(t-t^{\star}, \eta\right)
$$

Inequality 13, p 9 and the definition 15, p.10 imply

$$
\int_{0}^{s} \psi(v(t-\tau)) d \tau-\int_{0}^{s} \varphi^{\star}(u(\tau)) d \tau \leq-\left\langle\frac{1}{s} \int_{t-s}^{t} v(\tau) d \tau, \widehat{u}(s)\right\rangle
$$

Piecing these inequalities together and taking $s=t^{\star}$, we infer that

$$
\left\{\begin{array}{l}
\mathbf{c}(t, \xi)+\varepsilon+\left\langle\frac{1}{t^{\star}} \int_{t-t^{\star}}^{t} v(\tau) d \tau, \eta-\xi\right\rangle \\
\leq \mathbf{N}(t, \xi)+\int_{0}^{t^{\star}} \varphi^{\star}(u(\tau)) d \tau-\int_{0}^{t^{\star}} \psi(v(t-\tau)) d \tau \leq \mathbf{c}\left(t-t^{\star}, \eta\right)
\end{array}\right.
$$

from which we deduce that

$$
\varepsilon \leq \mathbf{c}\left(t-t^{\star}, \eta\right)-\mathbf{c}(t, \xi)-\left\langle\frac{1}{t^{\star}} \int_{t-t^{\star}}^{t} v(\tau) d \tau, \eta-\xi\right\rangle
$$

Consistency conditions [1], p 5 can be written in the form

$$
\forall 0 \leq r \leq s, \forall x \in K, y \in \partial K, \mathbf{c}(r, x)-\mathbf{c}(s, y) \leq\left\langle\frac{1}{s-r} \int_{r}^{s} v(\tau) d \tau, x-y\right\rangle
$$

Taking $r:=t-t^{\star}, s:=t, x:=\eta$ and $y:=\xi$, we obtain the contradiction $\varepsilon \leq 0$, and thus, we proved that for any $(t, \xi) \in \operatorname{Dom}(\mathbf{c}), \mathbf{N}(t, \xi)=\mathbf{c}(t, \xi)$.

\section{Other Auxiliary Systems}

For proving that the viability hyposolution is the solution in a generalized sense to the Hamilton-Jacobi partial differential equation derived from the tangential or normal conditions characterizing capture basins, we need assumptions that control system (8), $\mathrm{p}$ 6 does not satisfy. It happens that the capture basin of the hypograph of $\mathbf{c}$ viable in the hypograph of $\mathbf{b}$ under control system (8) is still the capture basin under other auxiliary systems which satisfy these assumptions, so that we shall be able to transfer the theorems concerning capture basins. 
The function $\psi$ being concave and finite, it is then continuous, so that, the function $v(\cdot)$ being bounded, the constant

$$
\alpha:=\sup _{u \in \operatorname{Dom}\left(\varphi^{\star}\right)} \varphi^{\star}(u)-\inf _{\tau \geq 0} \psi(v(\tau))
$$

is finite by Lemma 6.3. $\mathrm{p} 17$ below. The new characteristic control systems are defined by

$$
\left\{\begin{array}{lll}
\tau^{\prime}(t) & =-1 & \\
x^{\prime}(t)=u(t) & \text { where } u(t) \in \operatorname{Dom}\left(\varphi^{\star}\right) \\
y^{\prime}(t)=-\psi(v(\tau(t)))+\varphi^{\star}(u(t))+\pi(t) & \text { where } \pi(t) \in\left[0, \alpha+\psi\left(v(\tau(t))-\varphi^{\star}(u(t))\right]\right.
\end{array}\right.
$$

and

$$
\left\{\begin{array}{lll}
\tau^{\prime}(t) & =-1 & \\
x^{\prime}(t)=u(t) & \text { where } u(t) \in \operatorname{Dom}\left(\varphi^{\star}\right) \\
y^{\prime}(t)=-\psi(v(\tau(t)))+\varphi^{\star}(u(t))+\pi(t) & & \text { where } \pi(t) \geq 0
\end{array}\right.
$$

where we added a new control $\pi$ ranging in different intervals.

Lemma 6.1 Equality between Capture Basins. The capture basins of the hypograph of the function $\mathbf{c}$ by systems (8), 18) and (19) coincide:

$$
\operatorname{Capt}_{[19]}(\mathcal{H} y p(\mathbf{b}), \mathcal{H} y p(\mathbf{c}))=\operatorname{Capt}_{[18}(\mathcal{H} y p(\mathbf{b}), \mathcal{H} y p(\mathbf{c}))=\operatorname{Capt}_{\sqrt{8}}(\mathcal{H} y p(\mathbf{b}), \mathcal{H} y p(\mathbf{c}))
$$

Furthermore,

$$
\operatorname{Capt}_{[18)}(\mathcal{H} y p(\mathbf{b}), \mathcal{H} y p(\mathbf{c}))=\operatorname{Capt}_{[18\}}(\mathcal{H} y p(\mathbf{b}), \mathcal{H} y p(\mathbf{c}))-\{0\} \times\{0\} \times \mathbb{R}_{+}
$$

Proof - Inclusions

$$
\operatorname{Capt}_{\sqrt{8}}(\mathcal{H} y p(\mathbf{b}), \mathcal{H} y p(\mathbf{c})) \subset \operatorname{Capt}_{\sqrt[18]{ }}(\mathcal{H} y p(\mathbf{b}), \mathcal{H} y p(\mathbf{c})) \subset \operatorname{Capt}_{19}(\mathcal{H} y p(\mathbf{b}), \mathcal{H} y p(\mathbf{c}))
$$

are obvious. For proving that

$$
\operatorname{Capt}_{19}(\mathcal{H} y p(\mathbf{b}), \mathcal{H} y p(\mathbf{c})) \subset \operatorname{Capt}_{8}(\mathcal{H} y p(\mathbf{b}), \mathcal{H} y p(\mathbf{c}))
$$

let us consider an element $(t, x, y) \in \operatorname{Capt}_{19 p}(\mathcal{H} y p(\mathbf{b}), \mathcal{H} y p(\mathbf{c}))$. Hence there exist $u(\cdot) \in$ $L^{1}\left(0,+\infty ; \operatorname{Dom}\left(\varphi^{\star}\right)\right)$ and a corresponding solution $(\vec{\tau}(\cdot), x(\cdot), y(\cdot))$ to the characteristic control system 19 . starting from $(t, x, y)$ is given by $\tau(s)=t-s, x(s)=x+\int_{0}^{s} u(r) d r$ and

$$
y(s) \geq y-\int_{0}^{s}\left(\psi(v(t-r))-\varphi^{\star}(u(r))\right) d r
$$

and there exists $t^{\star} \in[0, t]$ such that $\left(t-t^{\star}, x\left(t^{\star}\right), y\left(t^{\star}\right)\right) \in \mathcal{H} y p(\mathbf{c})$ and, for all $s \in\left[0, t^{\star}\right],(t-s, x(s), y(s)) \in$ $\mathcal{H} y p(\mathbf{b})$. Setting

$$
y_{0}(s):=y+\int_{0}^{s}\left(\varphi^{\star}(u(r))-\psi(v(t-r))\right) d r
$$

we infer that $\left(\tau(\cdot), x(\cdot), y_{0}(\cdot)\right)$ is a solution to the to the characteristic control system 8 starting from $(t, x, y)$ viable in the environment $\mathcal{H} y p($ b) because 


$$
\forall s \in\left[0, t^{\star}\right], \quad y_{0}(s) \leq y(s) \leq \mathbf{b}(t-s, x(s))
$$

until time $t^{\star}$ where it reaches the target $\mathcal{H} y p(\mathbf{c})$ because

$$
y_{0}\left(t^{\star}\right) \leq y\left(t^{\star}\right) \leq \mathbf{c}\left(t-t^{\star}, x\left(t^{\star}\right)\right)
$$

This means that $(t, x, y) \in \operatorname{Capt}_{8 p}(\mathcal{H} y p(\mathbf{b}), \mathcal{H} y p(\mathbf{c}))$.

We also observe that whenever let us consider an element $(t, x, y) \in \operatorname{Capt} \sqrt{19 p}(\mathcal{H} y p(\mathbf{b}), \mathcal{H} y p(\mathbf{c}))$ and $z \leq y$, $(t, x, z)$ also belongs to the capture basin, so that,

$$
\operatorname{Capt}_{18\}}(\mathcal{H} y p(\mathbf{b}), \mathcal{H} y p(\mathbf{c}))=\operatorname{Capt}_{188}(\mathcal{H} y p(\mathbf{b}), \mathcal{H} y p(\mathbf{c}))-\{0\} \times\{0\} \times \mathbb{R}_{+}
$$

The proof is completed.

We also need

Lemma 6.2 Let $\psi: X \mapsto \mathbb{R}$ be an upper semicontinuous concave function.

The domain of its Fenchel transform $\varphi^{\star}$ is contained in a closed convex subset $A$ if and only if the function $\psi$ satisfies inequality

$$
\exists \beta \in \mathbb{R} \text { such that } \forall v \in X, \beta-\sigma_{A}(v) \leq \psi(v)
$$

Its Fenchel transform $\varphi^{\star}$ is bounded on a convex subset $A$ if and only if the function $\psi$ satisfies

$$
\exists \delta \in \mathbb{R} \text { such that } \forall v \in X^{\star}, \psi(v) \leq \delta-\sigma_{A}(v)
$$

Proof - Since $\psi(0)=\inf _{u \in \operatorname{Dom}\left(\varphi^{\star}\right)} \varphi^{\star}(u)$, we infer that

$$
\forall v \in X, \quad \forall u \in \operatorname{Dom}\left(\varphi^{\star}\right), \quad \psi(0)-\sigma_{\operatorname{Dom}\left(\varphi^{\star}\right)}(v) \leq \varphi^{\star}(u)-\langle u, v\rangle
$$

so that, by taking the infimum over $u$, we obtain inequality $\psi(0)-\sigma_{\operatorname{Dom}\left(\varphi^{\star}\right)}(v) \leq \psi(v)$. It is enough to set $\beta:=\psi(0)$ and to take $A:=\operatorname{Dom}\left(\varphi^{\star}\right)$. Conversely, assume that $\forall v \in X, \quad \psi(v) \geq \beta-\sigma_{A}(v)$. We shall prove that $\operatorname{Dom}\left(\varphi^{\star}\right) \subset A$. If not, there would exist $u \in \operatorname{Dom}\left(\varphi^{\star}\right) \backslash A$. The Separation Theorem states there exist $p_{0} \in X$ and $\varepsilon>0$ such that $\varepsilon \leq\left\langle p_{0}, u\right\rangle-\sigma_{A}\left(p_{0}\right)$. Consequently, for every $\lambda>0$,

$$
\lambda \varepsilon \leq\left\langle\lambda p_{0}, u\right\rangle-\sigma_{A}\left(\lambda p_{0}\right) \leq\left\langle\lambda p_{0}, u\right\rangle+\psi\left(\lambda p_{0}\right)-\beta \leq \varphi^{\star}(u)-\beta
$$

by assumption and by the definition of $\varphi^{\star}$. Letting $\lambda \mapsto+\infty$ implies that $\varphi^{\star}(u)=+\infty$, i.e., that $u \notin$ $\operatorname{Dom}\left(\varphi^{\star}\right)$, a contradiction.

For proving the second statement, we observe that if $\delta:=\sup _{u \in \operatorname{Dom}\left(\varphi^{\star}\right)} \varphi^{\star}(u)<+\infty$ is finite, then

$$
\psi(v) \leq \delta+\inf _{u \in \operatorname{Dom}\left(\varphi^{\star}\right)}\langle v,-u\rangle=\delta-\sigma_{\operatorname{Dom}\left(\varphi^{\star}\right)}(v)
$$

so that the inequality holds true with $A:=\operatorname{Dom}\left(\varphi^{\star}\right)$. Conversely, inequality $\psi(v) \leq \delta-\sigma_{A}(v)$ implies that

$$
\forall u \in A, \varphi^{\star}(u) \leq \sup _{v \in \operatorname{Dom}(\psi)}\left[\langle v, u\rangle+\delta-\sigma_{A}(v)\right]=c
$$

is bounded on $A$, and thus, on $\operatorname{Dom}\left(\varphi^{\star}\right)$ whenever this domain is contained in $A$. 
Control systems 18$)$ and $19, \mathrm{p} 15$ are actually differential inclusions

$$
\left(\tau^{\prime}(t), x^{\prime}(t), y^{\prime}(t)\right) \in F(\tau(t), x(t), y(t))
$$

where

$$
\begin{gathered}
F(\tau, x, y):=\left\{\left(-1, u,-\psi(v(\tau))+\varphi^{\star}(u)+\pi\right)\right\}_{u \in \operatorname{Dom}\left(\varphi^{\star}\right), \pi \in\left[0, \alpha+\psi(v(\tau))-\varphi^{\star}(u)\right]} \\
\left(\tau^{\prime}(t), x^{\prime}(t), y^{\prime}(t)\right) \in F_{\infty}(\tau(t), x(t), y(t))
\end{gathered}
$$

and where

$$
F_{\infty}(\tau, x, y):=\left\{\left(-1, u,-\psi(v(\tau))+\varphi^{\star}(u)+\pi\right)\right\}_{u \in \operatorname{Dom}\left(\varphi^{\star}\right), \pi \geq 0}
$$

Lemma 6.3 The set-valued map $F$ is Marchaud and, if the functions $\psi, \varphi^{\star}$ and $v$ are Lipschitz, the setvalued map $F_{\infty}$ is Lipschitz with closed images.

Proof - For proving that the set-valued map $F$ is Marchaud, we shall check successively that:

1. The values $F(\tau, x, y)$ of the set-valued map $F$ are convex: Indeed, for convex weight $\lambda_{i} \geq 0$ such that $\sum \lambda_{i}=1$, we can write

$$
\sum \lambda_{i}\left(-1, u_{i},-\psi(v(\tau))+\varphi^{\star}\left(u_{i}\right)+\pi_{i}\right)=\left(-1, \bar{u}, \varphi^{\star}(\bar{u})-\psi(v(\tau))+\bar{\pi}\right)
$$

where $\bar{u}:=\sum \lambda_{i} u_{i}$ and

$$
\bar{\pi}:=\sum \lambda_{i} \varphi^{\star}\left(u_{i}\right)-\varphi^{\star}\left(\sum \lambda_{i} u_{i}\right)+\sum \lambda_{i} \pi_{i}
$$

Since the domain of $\varphi^{\star}$ is convex, $\bar{u} \in \operatorname{Dom}\left(\varphi^{\star}\right)$. We observe that $\bar{\pi}$ is non negative and smaller than or equal to $\alpha+\psi(v(\tau))-\varphi^{\star}(\bar{u})$ because

$$
\left\{\begin{array}{l}
\bar{\pi} \leq \sum \lambda_{i} \varphi^{\star}\left(u_{i}\right)-\varphi^{\star}\left(\sum \lambda_{i} u_{i}\right)+\sum \lambda_{i}\left(\alpha+\psi(v(\tau))-\varphi^{\star}\left(u_{i}\right)\right) \\
=\alpha+\psi(v(\tau))-\varphi^{\star}\left(\sum \lambda_{i} u_{i}\right)
\end{array}\right.
$$

2. The graph of the set-valued map $F$ is closed: Indeed, let us consider a sequence of elements $\left(\left(\tau_{n}, x_{n}, y_{n}\right),\left(-1, u_{n}, \lambda_{n}\right)\right)$ of the graph of $F$ converging to $((\tau, x, y),(-1, u, \lambda))$ where $\lambda_{n}:=-\psi\left(v\left(\tau_{n}\right)\right)+$ $\varphi^{\star}\left(u_{n}\right)+\pi_{n}$ and where $\pi_{n} \in\left[0, \alpha+\psi\left(v\left(\tau_{n}\right)\right)-\varphi^{\star}\left(u_{n}\right)\right]$.

Since the function $(\tau, x, y, u) \mapsto \varphi^{\star}(u)-\psi(v(\tau))$ is lower semicontinuous and since

$$
\left(\tau_{n}, x_{n}, y_{n}, u_{n}, \lambda_{n}\right)=\left(\tau_{n}, x_{n}, y_{n}, u_{n},-\psi\left(v\left(\tau_{n}\right)\right)+\varphi^{\star}\left(u_{n}\right)+\pi_{n}\right)
$$

belongs to the epigraph of this function (because $\pi_{n}$ is positive by construction) which is closed, we deduce that the limit $(\tau, x, y, u, \lambda)$ also belongs to this epigraph, i.e., that $\lambda \geq \varphi^{\star}(u)-\psi(v(\tau))$. It is enough to set $\pi:=\lambda-\varphi^{\star}(u)-\psi(v(\tau)) \geq 0$, which from now on defines $\pi$. Recall that $\pi_{n}=$ $\lambda_{n}+\psi\left(v\left(\tau_{n}\right)\right)-\varphi^{\star}\left(u_{n}\right) \leq \alpha+\mathbf{l}\left(\tau_{n}, x_{n}\right)-\varphi^{\star}\left(u_{n}\right)$ by construction of $\pi_{n}$. Therefore, $\lambda_{n} \leq \alpha$. Therefore, taking the limit, $\lambda=\pi+\varphi^{\star}(u)-\psi(v(\tau)) \leq \alpha$. In summary, the limit $((\tau, x, y),(-1, u, \lambda))$ of elements $\left(\left(\tau_{n}, x_{n}, y_{n}\right),\left(-1, u_{n}, \lambda_{n}\right)\right)$ belongs to the graph of $F$ since $\lambda=-\psi(v(\tau))+\varphi^{\star}(u)+\pi$ where $\pi \in$ $\left[0, \alpha+\psi(v(\tau))-\varphi^{\star}(u)\right]$. 
3. The images $F(\tau, x, y)$ of $F$ are bounded: This follows from Lemma 6.2, $\mathrm{p} 16$ because $\operatorname{Dom}\left(\varphi^{\star}\right)$ is bounded and

$$
\varphi^{\star}(u)-\psi(v(\tau))+\pi \leq \alpha:=\sup _{u \in \operatorname{Dom}\left(\varphi^{\star}\right)} \varphi^{\star}(u)-\inf _{\tau \geq 0} \psi(v(\tau))
$$

is finite since $\varphi^{\star}$ is bounded above. Therefore

$$
\left\|\left(-1, u, \varphi^{\star}(u)-\psi(v(\tau))+\pi\right)\right\| \leq \max \left(1,\left\|\operatorname{Dom}\left(\varphi^{\star}\right)\right\|, \alpha\right)
$$

Hence, we have proved that the set-valued map $F$ is Marchaud. The Lipschitziannity of $F_{\infty}$ is obvious.

We thus deduce that

Proposition 6.4 Upper semicontinuity of the Solution. The viability hyposolution is upper semicontinuous and its hypograph satisfies:

$$
\mathcal{H} y p(\mathbf{N})=\operatorname{Capt}_{188}(\mathcal{H} y p(\mathbf{b}), \mathcal{H} y p(\mathbf{c}))=\operatorname{Capt}_{19]}(\mathcal{H} y p(\mathbf{b}), \mathcal{H} y p(\mathbf{c}))
$$

The viability hyposolution is concave whenever the functions $\mathbf{b}$ and $\mathbf{c}$ are concave.

Proof - The first statement follows from Proposition 4.3 of [5] stating that under a Marchaud control system, the capture basin of a target is closed whenever the target $\mathcal{H} y p(\mathbf{c})$ and the environment $\mathcal{H} y p(\mathbf{b})$ are closed and the complement of the target in the environment is a repeller: This is the case because the first component of the system is $\tau^{\prime}(t)=-1$ implies that all solutions $(t-s, x(s), y(s))$ starting from any $(t, x, y)$ leave $\mathbb{R}_{+} \times X \times \mathbb{R}$, and thus, $\mathcal{H} y p(\mathbf{b}) \subset \mathbb{R}_{+} \times X \times \mathbb{R}$. Since we have proved that

$$
\operatorname{Capt}_{180}(\mathcal{H} y p(\mathbf{b}, \mathcal{H} y p(\mathbf{c})))=\operatorname{Capt}_{18\}}(\mathcal{H} y p(\mathbf{b}, \mathcal{H} y p(\mathbf{c})))-\{0\} \times\{0\} \times \mathbb{R}_{+}
$$

we infer that $\operatorname{Capt}_{18}(\mathcal{H} y p(\mathbf{b}, \mathcal{H} y p(\mathbf{c})))$ is an hypograph, and thus, the hypograph of the viability hyposolution.

\section{Contingent Frankowska Solution to the Hamilton-Jacobi Equa- tion}

We shall prove that the viability hyposolution to Hamilton-Jacobi equation 22, p 5 (see Definition 3.1 p 6 ) is the contingent Frankowska solution by characterizing them in terms of tangent cones and translating them in terms of contingent Frankowska hyposolutions. 
Theorem 7.1 Contingent Frankowska Solution. The viability hyposolution $\mathbf{N}$ is the largest upper semicontinuous solution satisfying

$$
\psi(v(t)) \geq \inf _{u \in \operatorname{Dom}\left(\varphi^{\star}\right)}\left(\varphi^{\star}(u)-D_{\downarrow} \mathbf{N}(t, x)(-1, u)\right)
$$

and the initial/Dirichlet conditions and the inequality constraints. If the functions $\psi, \varphi^{\star}$ and $v$ are furthermore Lipschitz, then $\mathbf{N}$ is the smallest upper semi continuous solution satisfying

1. If $\mathbf{N}(t, x)<\mathbf{b}(t, x)$, then

$$
\psi(v(t)) \leq \inf _{u \in \operatorname{Dom}\left(\varphi^{\star}\right)}\left(D_{\downarrow} \mathbf{N}(t, x)(1,-u)+\varphi^{\star}(u)\right)
$$

2. If $\mathbf{N}(t, x)=\mathbf{b}(t, x)$, then

$$
\psi(v(t)) \leq \inf _{\left\{u \mid \psi(v(t)) \leq D_{\downarrow} \mathbf{b}(t, x)(1,-u)+\varphi^{\star}(u)\right\}}\left(D_{\downarrow} \mathbf{N}(t, x)(1,-u)+\varphi^{\star}(u)\right)
$$

Proof - Observe first that

$$
(t, x, y) \in \mathcal{H} y p(\mathbf{N}) \backslash \mathcal{H} y p(\mathbf{c}) \text { if and only if } t>0, x \in \partial K \text { and } y \leq \mathbf{N}(t, x)
$$

Indeed, $\mathcal{H} y p(\mathbf{N}) \backslash \mathcal{H} y p(\mathbf{c})$ is the set of $(t, x, y)$ such that $\mathbf{c}(t, x)<y \leq \mathbf{N}(t, x)$. This is automatically satisfied when $t>0$ and $x \in \partial K$ whenever $y \leq \mathbf{N}(t, x)$ since in this case, $\mathbf{c}(t, x)=-\infty$. It is impossible otherwise since, by Theorem 5.1. $\mathrm{p} .13 . \mathbf{N}(t, x)=\mathbf{c}(t, x)$.

Theorem 4.6 of [7] states that since $F$ is Marchaud by Lemma 6.3, the capture basin is the largest closed subset between the hypograph of $\mathbf{c}$ and $\mathbb{R}_{+} \times X \times \mathbb{R}$ such that $\mathcal{H} y p(\mathbf{N}) \backslash \mathcal{H} y p(\mathbf{c})$ is locally viable under $F$.

Theorems 3.2.4 and 3.3.4 of [7] state that $\mathcal{H} y p(\mathbf{N}) \backslash \mathcal{H} y p(\mathbf{c})$ is locally viable under $F$ if and only if $\forall t>$ $0, \forall x \in X, \forall y \leq \mathbf{N}(t, x), \quad \exists u \in \operatorname{Dom}\left(\varphi^{\star}\right), \exists \pi \in\left[0, \alpha+\psi(v(\tau))-\varphi^{\star}(u)\right]$, such that

$$
\left(-1, u,-\psi(v(t))+\varphi^{\star}(u)+\pi\right) \in T_{\mathcal{H} y p(\mathbf{N})}(t, x, y)
$$

If $y=\mathbf{N}(t, x)$, then

$$
T_{\mathcal{H} y p(\mathbf{N})}(t, x, \mathbf{N}(t, x))=: \mathcal{H} y p\left(D_{\downarrow} \mathbf{N}(t, x)\right)
$$

so that we infer that there exists $u \in \operatorname{Dom}\left(\varphi^{\star}\right)$

$$
-\psi(v(t))+\varphi^{\star}(u)+\pi \leq D_{\downarrow} \mathbf{N}(t, x)(-1, u)
$$

from which inequality 22 ensues.

Conversely, since $D_{\downarrow} \mathbf{N}(t, x)(-1, \cdot)$ is upper semicontinuous and the support of $\varphi^{\star}$ is compact, inequality (22) implies the existence of $u \in \operatorname{Dom}\left(\varphi^{\star}\right)$ such that

$$
\left(-1, u,-\psi(v(t))+\varphi^{\star}(u)+\pi\right) \in T_{\mathcal{H} y p(\mathbf{N})}(t, x, \mathbf{N}(t, x))
$$

When $y<\mathbf{N}(t, x)$, then Lemma 9.10 implies that

$$
\left(-1, u,-\psi(v(t))+\varphi^{\star}(u)+\pi\right) \in T_{\mathcal{H} y p(\mathbf{N})}(t, x, y)
$$


because $(-1, u)$ belongs to the domain of $D_{\downarrow} \mathbf{N}(t, x)$.

By Theorems 4.7 and 4.10 of [7], the capture basin is the smallest closed subset between the hypographs of $\mathbf{c}$ and $\mathbf{c}$ such that $\mathcal{H} y p(\mathbf{N})$ is backward invariant with respect to $\mathcal{H} y p(\mathbf{b})$. Since $F_{\infty}$ is Lipschitz by Lemma 6.3 whenever the functions $\psi, \varphi^{\star}$ and $v$ are Lipschitz, the invariance theorem (Theorem 5.3.4 in [5]) states that $\mathcal{H} y p(\mathbf{N})$ is backward invariant with respect to $\mathcal{H} y p(\mathbf{b})$ under $F_{\infty}$ if and only if

$$
\forall(t, x, y) \in \mathcal{H} y p(\mathbf{N}), \quad F_{\infty}(t, x, y) \cap T_{\mathcal{H} y p(\mathbf{b})}(t, x, y) \subset T_{\mathcal{H} y p(\mathbf{N})}(t, x, y)
$$

Since the function $\mathbf{b}$ is assumed to be continuous,

$$
\operatorname{Int}(\mathcal{H} y p(\mathbf{N}))=\{(t, x, y) \text { such that } y<\mathbf{b}(t, x)\}
$$

Therefore, we have to investigate two cases:

1. either for all $(t, x, y) \in \mathcal{H} y p(\mathbf{N}) \cap \operatorname{Int}(\mathcal{H} y p(\mathbf{b}))$.

Then

$\forall t \geq 0, \forall x \in X, \forall y \leq \mathbf{N}(t, x), \quad \forall u \in \operatorname{Dom}\left(\varphi^{\star}\right), \forall \pi \in\left[0, \alpha+\psi(v(\tau))-\varphi^{\star}(u)\right]$,

$$
\left(1,-u, \psi(v(t))-\varphi^{\star}(u)-\pi\right) \in T_{\mathcal{H} y p(\mathbf{N})}(t, x, y)
$$

If $y=\mathbf{N}(t, x)$, then so that we infer that for all $u \in \operatorname{Dom}\left(\varphi^{\star}\right)$

$$
\psi(v(t))-\varphi^{\star}(u) \leq D_{\downarrow} \mathbf{N}(t, x)(1,-u)
$$

from which we derive inequality 23$)$. Conversely, since for all $u \in \operatorname{Dom}\left(\varphi^{\star}\right),(1,-u)$ belongs to the domain of $D_{\downarrow} \mathbf{N}(t, x)$, we derive that

$$
\left(1,-u, \psi(v(t))-\varphi^{\star}(u)-\pi\right) \in T_{\mathcal{H} y p(\mathbf{N})}(t, x, y)
$$

holds true.

2. or for all $(t, x, y) \in \mathcal{H} y p(\mathbf{N}) \cap \partial(\mathcal{H} y p(\mathbf{b}))$, and in this case, $y=\mathbf{N}(t, x)=\mathbf{b}(t, x)$. Then, $\forall t \geq 0, \forall x \in X, \forall u \in \operatorname{Dom}\left(\varphi^{\star}\right), \forall \pi \geq 0$ such that

$$
\left(1,-u, \psi(v(t))-\varphi^{\star}(u)-\pi\right) \in T_{\mathcal{H} y p(\mathbf{b})}(t, x, y)
$$

we have

$$
\left(1,-u, \psi(v(t))-\varphi^{\star}(u)-\pi\right) \in T_{\mathcal{H} y p(\mathbf{N})}(t, x, y)
$$

This means that whenever

$$
\psi(v(t)) \leq D_{\downarrow} \mathbf{b}(t, x)(1,-u)+\varphi^{\star}(u)
$$

then

which is 24, $\mathrm{p} 19$.

$$
\psi(v(t)) \leq D_{\downarrow} \mathbf{N}(t, x)(1,-u)+\varphi^{\star}(u)
$$


Theorem 4.5. $\mathrm{p} 11$ states that the viability hyposolution is the valuation function 16 , 11 of the underlying optimal control problem (8), $\mathrm{p} 6$.

The associated regulation map $R$ for regulating the optimal evolutions is thus defined by

$$
\forall t>0, x \in X, \quad R(t, x):=\left\{u \mid 0 \leq D_{\downarrow} \mathbf{N}(t, x)(-1, u)-\varphi^{\star}(u)+\psi(v(t))\right\}
$$

One can prove that the optimal solutions of the control problem are governed by the control system

$$
\left\{\begin{array}{l}
\tau^{\prime}(s)=-1 \\
x^{\prime}(s)=u(s) \in R(\tau(s), x(s)) \\
y^{\prime}(s)=\varphi^{\star}(u(s))-\psi(v(\tau(s)))
\end{array}\right.
$$

This motivates a further study of the regulation map. If the solution $\mathbf{N}$ is differentiable, the regulation map can be written in the form

$$
R(t, x):=\left\{u \mid 0 \leq-\frac{\partial \mathbf{N}(t, x)}{\partial t}+\frac{\partial \mathbf{N}(t, x)}{\partial x} u-\varphi^{\star}(u)+\psi(v(t))\right\}
$$

The elements $u$ maximizing the right-hand side are the elements belonging to $-\partial_{+} \psi\left(\frac{\partial \mathbf{N}(t, x)}{\partial x}\right)$, Consequently,

$$
-\partial_{+} \psi\left(\frac{\partial \mathbf{N}(t, x)}{\partial x}\right) \subset R(t, x)
$$

Actually, approximations of the regulation map and thus, optimal evolutions, as well as the solution to the Hamilton-Jacobi-Bellman equation are provided by the Capture Basin Algorithm.

\section{Barron-Jensen/Frankowska Solution to the Hamilton-Jacobi Equation}

Instead of characterizing capture basins in terms of tangent cones and translating them in terms of contingent Frankowska hyposolutions, we translate them in the equivalent formulation of Frankowska-Barron/Jensen solutions, a weaker concept of viscosity solutions requiring only the upper semicontinuity of the solution instead of its continuity. 
Theorem 8.1 Barron-Jensen/Frankowska Solution. The viability hyposolution $\mathbf{N}$ is the largest upper semicontinuous solution between $\mathbf{c}$ and $\mathbf{b}$ satisfying

$$
\left\{\begin{array}{l}
\text { (i) } \quad \forall t>0, \forall x \in \operatorname{Int}(K), \forall\left(p_{t}, p_{x}\right) \in \partial_{+} \mathbf{N}(t, x), p_{t}+\psi\left(p_{x}\right) \leq \psi(v(t)) \\
\text { (ii) } \quad \forall t>0, \forall x \in \operatorname{Int}(K), \forall\left(p_{t}, p_{x}\right) \in\left(\operatorname{Dom}\left(D_{\downarrow} \mathbf{N}(t, x)\right)\right)^{-}, \quad p_{t}-\sigma\left(\operatorname{Dom}\left(\varphi^{\star}\right), p_{x}\right) \leq 0
\end{array}\right.
$$

If the functions $\psi, \varphi^{\star}$ and $v$ are furthermore Lipschitz, then $\mathbf{N}$ is the smallest upper semi continuous solution between $\mathbf{c}$ and $\mathbf{b}$ satisfying

1. If $\mathbf{N}(t, x)<\mathbf{b}(t, x)$, then

$$
\begin{cases}\text { (i) } \quad & \forall t \geq 0, \forall x \in K \text { such that } \mathbf{N}(t, x)<\mathbf{b}(t, x), \forall\left(p_{t}, p_{x}\right) \in \partial_{+} \mathbf{N}(t, x) \\ & p_{t}+\psi\left(p_{x}\right) \geq \psi(v(t)) \\ \text { (ii }) \quad & \forall t \geq 0, \forall x \in K \text { such that } \mathbf{N}(t, x)<\mathbf{b}(t, x), \forall\left(p_{t}, p_{x}\right) \in\left(\operatorname{Dom}\left(D_{\downarrow} \mathbf{N}(t, x)\right)\right)^{-} \\ & p_{t}-\sigma\left(\operatorname{Dom}\left(\varphi^{\star}\right), p_{x}\right) \geq 0\end{cases}
$$

2. If $\mathbf{N}(t, x)=\mathbf{b}(t, x)$, then

$$
\begin{cases} & \forall\left(p_{t}, p_{x}\right) \in \partial_{+} \mathbf{N}(t, x), \quad \exists\left(q_{t}, q_{x}\right) \in \partial_{+} \mathbf{b}(t, x) \text { and } 0<\mu<1 \text { such that } \\ \text { either } & p_{t}-q_{t}-\sigma\left(\operatorname{Dom}\left(\varphi^{\star}\right), p_{x}-q_{x}\right) \geq 0 \\ \text { or } & \frac{p_{t}-\mu q_{t}}{1-\mu}+\psi\left(\frac{p_{x}-\mu q_{x}}{1-\mu}\right) \geq \psi(v(t))\end{cases}
$$

and thus, the unique upper semicontinuous solution satisfying all these properties.

Observe that under the Lipschitz assumptions, the hypo viability solution satisfies

$$
\left\{\begin{array}{l}
\quad \forall t>0, \forall x \in \operatorname{Int}(K) \text { such that } \mathbf{N}(t, x)<\mathbf{b}(t, x) \\
\text { (i) } \quad \forall\left(p_{t}, p_{x}\right) \in \partial_{+} \mathbf{N}(t, x), p_{t}+\psi\left(p_{x}\right)=\psi(v(t)) \\
(i i) \quad \forall\left(p_{t}, p_{x}\right) \in\left(\operatorname{Dom}\left(D_{\downarrow} \mathbf{N}(t, x)\right)\right)^{-}, p_{t}-\sigma\left(\operatorname{Dom}\left(\varphi^{\star}\right), p_{x}\right)=0
\end{array}\right.
$$

Proof - Proposition 6.4 p 18 states that the hypograph of the viability hyposolution satisfies:

$$
\mathcal{H} y p(\mathbf{N})=\operatorname{Capt}_{18}(\mathcal{H} y p(\mathbf{b}), \mathcal{H} y p(\mathbf{c}))=\operatorname{Capt}_{19 p}(\mathcal{H} y p(\mathbf{b}), \mathcal{H} y p(\mathbf{c}))
$$

Theorem 9.4, p 26 states that $\operatorname{Capt}_{\sqrt{18})}(\mathcal{H} y p(\mathbf{b}), \mathcal{H} y p(\mathbf{c}))$ is the largest subset $\mathcal{D}$ between $\mathcal{C}$ and $\mathcal{K}$ such that $\mathcal{D} \backslash \mathcal{C}$ is locally invariant.

Taking $\mathcal{D}:=\mathcal{H} y p(\mathbf{N})$, Theorems 3.2.4 and 3.3.4 of [5] state that $\mathcal{H} y p(\mathbf{N}) \backslash \mathcal{H} y p(\mathbf{c})$ is locally viable under $F$ if and only if $\forall t>0, \forall x \in \operatorname{Int}(K), \forall y \leq \mathbf{N}(t, x), \quad \exists u \in \operatorname{Dom}\left(\varphi^{\star}\right), \exists \pi \in\left[0, \alpha+\psi(v(t))-\varphi^{\star}(u)\right]$, such that $\forall\left(-p_{t},-p_{x}, \lambda\right) \in N_{\mathcal{H} y p(\mathbf{N})}(t, x, y)$,

$$
\left\{\begin{array}{l}
\left\langle\left(-p_{t},-p_{x}, \lambda\right),\left(-1, u,-\psi(v(t))+\varphi^{\star}(u)+\pi\right)\right\rangle \\
=p_{t}-\left\langle p_{x}, u\right\rangle+\lambda\left(-\psi(v(t))+\varphi^{\star}(u)+\pi\right) \leq 0
\end{array}\right.
$$

By Lemma 9.10 if $y=\mathbf{N}(t, x),\left(-p_{t},-p_{x}, \lambda\right) \in N_{\mathcal{H} y p(\mathbf{N})}(t, x, y)$ means that either $\lambda>0$, and that, taking $\lambda=1,\left(p_{t}, p_{x}\right) \in \partial_{+} \mathbf{N}(t, x)$ or that $\lambda=0$, and that $\left(p_{t}, p_{x}\right) \in\left(\operatorname{Dom}\left(D_{\downarrow} \mathbf{N}(t, x)\right)\right)^{-}$. If $y<\mathbf{N}(t, x)$, $\left(-p_{t},-p_{x}, \lambda\right) \in N_{\mathcal{H} y p(\mathbf{N})}(t, x, y)$ means also that $\lambda=0$, and that $\left(p_{t}, p_{x}\right) \in\left(\operatorname{Dom}\left(D_{\downarrow} \mathbf{N}(t, x)\right)\right)^{-}$.

Consequently, condition 29 can be written in the following form: 
- Case when $y=\mathbf{N}(t, x)$ and $\lambda=1$ :

$$
\left\{\begin{array}{l}
\forall t>0, \forall x \in \operatorname{Int}(K), \forall\left(p_{t}, p_{x}\right) \in \partial_{+} \mathbf{N}(t, x), \text { then } \\
p_{t}-\psi(v(t))+\inf _{u \in \operatorname{Dom}\left(\varphi^{\star}\right)}\left[\varphi^{\star}(u)-\left\langle p_{x}, u\right\rangle\right] \\
=p_{t}-\psi(v(t))+\psi\left(p_{x}\right) \leq 0
\end{array}\right.
$$

- Case when $y \leq \mathbf{N}(t, x)$ and $\lambda=0$ :

$$
\left\{\begin{array}{l}
\forall t>0, \forall x \in \operatorname{Int}(K), \forall\left(p_{t}, p_{x}\right) \in\left(\operatorname{Dom}\left(D_{\downarrow} \mathbf{N}(t, x)\right)\right)^{-}, \text {then } \\
p_{t}-\sup _{u \in \operatorname{Dom}\left(\varphi^{\star}\right)}\left\langle p_{x}, u\right\rangle=p_{t}-\sigma\left(\operatorname{Dom}\left(\varphi^{\star}\right), p_{x}\right) \leq 0
\end{array}\right.
$$

(Recall that this condition disappears whenever the viability hyposolution $\mathbf{N}$ is hypo-differentiable, and, in particular, when the hyposolution is Lipschitz).

Proof of inequalities 28) and (27): Theorem 9.4 p 26 states that $\operatorname{Capt}_{19 \mathrm{p}}(\mathcal{H} y p(\mathbf{b}), \mathcal{H} y p(\mathbf{c}))$ is the smallest subset $\mathcal{D}$ between $\mathcal{C}$ and $\mathcal{K}$ such that $\mathcal{D}$ is backward invariant with respect to $\mathcal{K}$. Theorem 9.7, p.27 and Lemma 9.8, $\mathrm{p} 27$ states that $\mathcal{D}:=\mathcal{H} y p(\mathbf{N})$ is backward invariant with respect to $\mathcal{H} y p(\mathbf{b})$ under 19 if and only if

1. either for all $(t, x, y) \in \mathcal{H} y p(\mathbf{N}) \cap \operatorname{Int}(\mathcal{H} y p(\mathbf{N}))$,

$$
\forall\left(-p_{t}-p_{x}, \lambda\right) \in N_{\mathcal{H} y p(\mathbf{N})}(t, x, y), \quad \sigma\left(F_{\infty}(x),\left(p_{t}, p_{x},-\lambda\right)\right) \leq 0
$$

Since the function $\mathbf{b}$ is assumed to be continuous,

$$
\operatorname{Int}(\mathcal{H} y p(\mathbf{N}))=\{(t, x, y) \text { such that } y<\mathbf{b}(t, x)\}
$$

the first case means that $y \leq \mathbf{N}(t, x)<\mathbf{b}(t, x)$ and the above condition that

$$
\left\{\begin{array}{l}
\forall\left(-p_{t}-p_{x}, \lambda\right) \in N_{\mathcal{H} y p(\mathbf{N})}(t, x, y) \\
=-p_{t}+\left\langle p_{x}, u\right\rangle+\lambda\left(\psi(v(t))-\varphi^{\star}(u)-\pi\right) \leq 0
\end{array}\right.
$$

This implies that $\lambda \geq 0$.

Consequently, condition 30 can be written in the following form:

- Case when $y=\mathbf{N}(t, x)<\mathbf{b}(t, x)$ and $\lambda=1$ :

$$
\left\{\begin{array}{l}
\forall t>0, \forall x \in X, \forall\left(p_{t}, p_{x}\right) \in \partial_{+} \mathbf{N}(t, x), \text { then } \\
-p_{t}+\psi(v(t))+\sup _{u \in \operatorname{Dom}\left(\varphi^{\star}\right)}\left[\left\langle p_{x}, u\right\rangle-\varphi^{\star}(u)\right] \\
=-p_{t}+\psi(v(t))-\psi\left(p_{x}\right) \leq 0
\end{array}\right.
$$

- Case when $y \leq \mathbf{N}(t, x)$ and $\lambda=0$ :

$$
\left\{\begin{array}{l}
\forall t>0, \forall x \in X, \forall\left(p_{t}, p_{x}\right) \in\left(\operatorname{Dom}\left(D_{\downarrow} \mathbf{N}(t, x)\right)\right)^{-}, \text {then } \\
-p_{t}+\sup _{u \in \operatorname{Dom}\left(\varphi^{\star}\right)}\left\langle p_{x}, u\right\rangle=-p_{t}+\sigma\left(\operatorname{Dom}\left(\varphi^{\star}\right), p_{x}\right) \leq 0
\end{array}\right.
$$

- Case when $y=\mathbf{N}(t, x)=\mathbf{b}(t, x)$. 
2. or for all $(t, x, y) \in \mathcal{H} y p(\mathbf{N}) \cap \partial(\mathcal{H} y p(\mathbf{N}))$, and in this case, $y=\mathbf{N}(t, x)=\mathbf{b}(t, x)$ and

$\forall\left(-p_{t}-p_{x}, \lambda\right) \in N_{\mathcal{H} y p(\mathbf{N})}(t, x, y), \quad \exists\left(-q_{t}-q_{x}, \mu\right) \in N_{\mathcal{H} y p(\mathbf{b})}(t, x, y) \quad$ such that $\sigma\left(F_{\infty}(x),\left(p_{t}-q_{t}, p_{x}-q_{x}, \mu-\lambda\right)\right) \leq 0$

where $\lambda \geq 0$ and $\mu>0$ since we have assumed that $\mathbf{b}$ is Lipschitz, and thus hypodifferentiable. This can be translated in the following form

$$
-p_{t}+q_{t}+\sup _{u}\left(\left\langle p_{x}-q_{x}, u\right\rangle+(\mu-\lambda)\left(\varphi^{\star}(u)\right)+\sup _{\pi \geq 0}(\mu-\lambda) \pi-\psi(v(t))\right) \leq 0
$$

This implies that $\lambda \geq \mu>0$.

- case when $\lambda-\mu=0$. It happens when both $\left(p_{t}, p_{x}\right) \in \partial_{+} \mathbf{N}(t, x)$ and $\left(q_{t}, q_{x}\right) \in \partial_{+} \mathbf{b}(t, x)$. In this case, the above inequality boils down to

$$
-p_{t}+q_{t}+\sigma\left(\operatorname{Dom}\left(\varphi^{\star}\right), p_{x}-q_{x}\right) \leq 0
$$

- case when $\lambda-\mu>0$. The condition states that for every $\lambda>0$ and $\left(p_{t}, p_{x}\right) \in \partial_{+} \mathbf{N}(t, x)$, there exist $0<\mu<\lambda$ and $\left(q_{t}, q_{x}\right) \in \partial_{+} \mathbf{b}(t, x)$ such that

$$
-\frac{\lambda p_{t}-\mu q_{t}}{\lambda-\mu}+\sup _{u}\left(\left\langle\frac{\lambda p_{x}-\mu q_{x}}{\lambda-\mu}, u\right\rangle-\varphi^{\star}(u)\right)+\psi(v(t)) \leq 0
$$

which can be written

$$
-\frac{\lambda p_{t}-\mu q_{t}}{\lambda-\mu}-\psi\left(\frac{\lambda p_{x}-\mu q_{x}}{\lambda-\mu}\right)+\psi(v(t)) \leq 0
$$

This completes the proof.

\section{Appendix}

\subsection{Some Prerequisites from Viability Theory}

Here, $X:=\mathbb{R}^{n}$ and $Y:=\mathbb{R}^{m}$ denote finite dimensional vector spaces. Let $f: X \times Y \mapsto X$ be a singlevalued map describing the dynamics of a control system and $U: X \leadsto Y$ the set-valued map describing the state-dependent constraints on the controls.

First, any solution to a control system with state-dependent constraints on the controls

$$
\begin{cases}(i) & x^{\prime}(t)=f(x(t), u(t)) \\ (i i) & u(t) \in U(x(t))\end{cases}
$$

can be regarded as a solution to the differential inclusion $x^{\prime}(t) \in F(x(t))$ where the right hand side is defined by $F(x):=f(x, U(x)):=\{f(x, u)\}_{u \in U(x)}$.

We denote by $\mathcal{S}(x) \subset \mathcal{C}(0, \infty ; X)$ the set of absolutely continuous functions $t \mapsto x(t) \in X$ satisfying

$$
\text { for almost all } t \geq 0, \quad x^{\prime}(t) \in F(x(t))
$$


starting at time 0 at $x: x(0)=x$. The set-valued map $\mathcal{S}: X \leadsto \mathcal{C}(0, \infty ; X)$ is called the solution map associated with $F$.

Therefore, from now on, as long as we do not need to implicate explicitly the controls in our study, we shall replace control problems by differential inclusions.

We shall say that $K$ is locally viable under $F$ if from every $x \in K$ starts a solution $x(\cdot)$ to the differential inclusion $x^{\prime} \in F(x)$ viable in $K$ on the nonempty interval $\left[0, T_{x}\right.$ [ in the sense

$$
\forall t \in\left[0, T_{x}[, \quad x(t) \in K\right.
$$

and that $K$ is viable if we can take $T_{x}=+\infty$. It is locally backward invariant under $F$ if for every $\left.t_{0} \in\right] 0,+\infty\left[, x \in K\right.$, for all solutions $x(\cdot)$ to the differential inclusion $x^{\prime} \in F(x)$ arriving at $x$ at time $t_{0}$, there exists $s \in\left[0, t_{0}\left[\operatorname{such}\right.\right.$ that $x(\cdot)$ is viable in $K$ on the interval $\left[s, t_{0}\right]$, and backward invariant if we can take $s=0$.

We denote by

$$
\operatorname{Graph}(F):=\{(x, y) \in X \in Y \mid y \in F(x)\}
$$

the graph of a set-valued map $F: X \leadsto Y$ and $\operatorname{Dom}(F):=\{x \in X \mid F(x) \neq \emptyset\}$ its domain.

Most of the results of viability theory are true whenever we assume that the dynamics is Marchaud:

Definition 9.1 Marchaud Map. We shall say that $F$ is a Marchaud map if

$$
\left\{\begin{array}{l}
(i) \quad \text { the graph of } F \text { is closed } \\
\text { (ii) } \text { the values } F(x) \text { of } F \text { are convex } \\
\text { (iii) the growth of } F \text { is linear: } \exists c>0 \mid \forall x \in X,\|F(x)\|:=\sup _{v \in F(x)}\|v\| \leq c(\|x\|+1)
\end{array}\right.
$$

We shall say that $F$ is $\lambda$-Lipschitz if

$$
\forall x, y \in X, F(x) \subset F(y)+\lambda\|x-y\| B
$$

where $B$ is the unit ball.

This covers the case of Marchaud control systems where $(x, u) \mapsto f(x, u)$ is continuous, affine with respect to the controls $u$ and with linear growth and when $U$ is Marchaud.

We recall the following version of the important Theorem 3.5.2 of Viability Theory, [5]:

Theorem 9.2 The Stability Theorem . Assume that $F: X \leadsto X$ is Marchaud. Then the solution map $\mathcal{S}$ is upper semicompact with nonempty values: This means that whenever $x_{n} \in X$ converge to $x$ in $X$ and $x_{n}(\cdot) \in \mathcal{S}\left(x_{n}\right)$ is a solution to the differential inclusion $x^{\prime} \in F(x)$ starting at $x_{n}$, there exists a subsequence (again denoted by) $x_{n}(\cdot)$ converging to a solution $x(\cdot) \in \mathcal{S}(x)$ uniformly on compact intervals.

We shall also need some other prerequisites from Viability Theory:

Definition 9.3 Capture Basin of a Target. Let $C \subset K \subset X$ be two subsets, $C$ being regarded as a target, $K$ as a constrained set. The subset $\operatorname{Capt}(K, C)$ of initial states $x_{0} \in K$ such that $C$ is reached in finite time before possibly leaving $K$ by at least one solution $x(\cdot) \in \mathcal{S}\left(x_{0}\right)$ starting at $x_{0}$ is called the viable-capture basin of $C$ in $K . A$ subset $K$ is a repeller under $F$ if all solutions starting from $K$ leave $K$ in finite time. $A$ subset $D$ is locally backward invariant relatively to $K$ if all backward solutions starting from $D$ viable in $K$ are actually viable in $K$. 
We recall the following result of $[9]$ :

Theorem 9.4 Fixed-Point Characterization of Capture Basins. The viable-capture basin $\operatorname{Capt}(K, C)$ of a target $C$ viable in $K$ is

1. the largest subset $D$ satisfying $C \subset D \subset K$ and $D \subset \operatorname{Capt}(D, C)$,

2. the smallest subset $D$ satisfying $C \subset D \subset K$ and $\operatorname{Capt}(K, D) \subset D$,

3. the unique subset $D$ satisfying $C \subset D \subset K$ and

$$
D=\operatorname{Capt}(K, D)=\operatorname{Capt}(D, C)
$$

The subset $K \backslash C$ denotes the intersection of $K$ and the complement of $C$, i.e., is the set of elements of $K$ which do not belong to $C$. We can derive the following characterization of capture basin (see [7):

Theorem 9.5 Viability Characterization of Capture Basins. Let us assume that $F$ is Marchaud and that the subsets $C \subset K$ and $K$ are closed. If $K \backslash C$ is a repeller (this is the case when $K$ itself is a repeller), then the viable-capture basin $\operatorname{Capt}(K, C)$ of the target $C$ under $\mathcal{S}$ is the unique closed subset satisfying $C \subset D \subset K$ and

$$
\begin{cases}(i) & D \backslash C \text { is locally viable under } \mathcal{S} \\ (\text { ii }) & D \text { is locally backward invariant relatively to } K\end{cases}
$$

The contingent cone $T_{L}(x)$ to $L \subset X$ at $x \in L$ is the set of directions $v \in X$ such that there exist sequences $h_{n}>0$ converging to 0 and $v_{n}$ converging to $v$ satisfying $x+h_{n} v_{n} \in K$ for every $n$ (see for instance [12] or [76] for more details). The (regular) normal cone is the polar cone $N_{L}(x):=\left(T_{L}(x)\right)^{-}$of the contingent cone. We introduce the following Frankowska property that we need for deriving the system of HamiltonJacobi-Bellman equations of which the detector is a solution:

Definition 9.6 Frankowska Property. Let us consider a set-valued map $F: X \leadsto X$ and two subsets $C \subset K$ and $K$. We shall say that a subset $D$ between $C$ and $K$ satisfies the Frankowska property with respect to $F$ if

$$
\begin{cases}\text { (i) } & \forall x \in D \backslash C, \quad F(x) \cap T_{D}(x) \neq \emptyset \\ \text { (ii) } & \forall x \in D \cap \operatorname{Int}(K),-F(x) \subset T_{D}(x) \\ \text { (iii) } & \forall x \in D \cap \partial K, \quad-F(x) \cap T_{K}(x) \subset T_{D}(x)\end{cases}
$$

Actually, conditions (32) (ii) and (iii), p 26 boil down to the same condition

$$
\forall x \in D,-F(x) \cap T_{K}(x) \subset T_{D}(x)
$$

When $K$ is assumed further to be backward locally invariant, the above conditions 32 boil down to

$$
\left\{\begin{array}{l}
\text { (i) } \quad \forall x \in D \backslash C, \quad F(x) \cap T_{D}(x) \neq \emptyset \\
\text { (ii) } \quad \forall x \in D,-F(x) \subset T_{D}(x)
\end{array}\right.
$$

Theorem 9.5 and the Viability 7 and Invariance Theorems imply

\footnotetext{
${ }^{7}$ See for instance Theorems 3.2.4, 3.3.2 and 3.5.2 of [5].
} 
Theorem 9.7 Tangential Characterization of Capture Basins. Let us assume that $F$ is Marchaud, that $K$ is closed and that a closed subset $C$ satisfies $\operatorname{Viab}_{F}(K \backslash C)=\emptyset$. Then the viable-capture basin $\operatorname{Capt}_{F}^{K}(C)$ is

1. the largest closed subset $D$ satisfying $C \subset D \subset K$ and

$$
\forall x \in D \backslash C, \quad F(x) \cap T_{D}(x) \neq \emptyset
$$

2. if $F$ is Lipschitz, the unique closed subset D satisfying the Frankowska property (32).

We provide the dual characterization of the capture basin in terms of normal cones due to Hélène Frankowska:

\section{Lemma 9.8 Normal Characterization of Capture Basins. Let us assume that for}

$$
\forall x \in K, \quad 0 \in \operatorname{Int}\left(F(x)+T_{K}(x)\right)
$$

Then the Frankowska property [32), $p 26$ is equivalent to the dual Frankowska property

$$
\begin{cases}\text { (i) } & \forall x \in D \backslash C, \forall x \in N_{D}(x), \sigma(F(x),-p) \geq 0 \\ \text { (ii }) & \forall x \in D \cap \operatorname{Int}(K), \forall x \in N_{D}(x), \quad \sigma(F(x),-p) \leq 0 \\ \text { (iii) } & \forall x \in D \cap \partial K, \forall p \in N_{D}(x), \quad \inf _{q \in N_{K}(x)} \sigma(F(x), q-p) \leq 0\end{cases}
$$

Proof - Whenever $0 \in \operatorname{Int}\left(F(x)+T_{K}(x)\right)$, Proposition 3.9, p.50 of [6] implies that the support function of $-F(x) \cap T_{K}(x)$ is the inf-convolution of the support functions of $-F(x)$ and $T_{K}(x)$ :

$$
\sigma\left(-F(x) \cap T_{K}(x), p\right)=\inf _{q \in N_{K}(x)} \sigma(F(x), q-p)
$$

Consequently, inclusion $-F(x) \cap T_{K}(x) \subset T_{D}(x)$ is equivalent to

$$
\forall p, \inf _{q \in N_{K}(x)} \sigma(F(x), q-p) \leq \sigma\left(T_{D}(x), p\right)
$$

which can be written

$$
\forall p \in N_{D}(x), \inf _{q \in N_{K}(x)} \sigma(F(x), q-p) \leq 0
$$

This concludes the proof.

\subsection{Some Prerequisites of Convex Analysis}

We gather in this section notations and some results on convex analysis for the convenience of the reader non familiar with this topic. Since the authors of most of books on convex analysis have chosen to study convex functions rather than concave ones, we have chosen to associate with the concave function $\psi$ the Fenchel transform $\varphi^{\star}$ of $\varphi:=-\psi$ rather than the "concave Fenchel" transform $\psi^{\bigotimes}$ defined by the concave function

$$
\psi^{\bigotimes}(u):=\inf _{p \in \operatorname{Dom}(\psi)}[\langle p, u\rangle-\psi(p)]=-\varphi^{\star}(-u)
$$

The basic theorem of convex analysis states that $\psi=\psi^{\bigotimes \bigotimes}$ if and only if $\psi$ is concave, upper semicontinuous, and non trivial (i.e. $\operatorname{Dom}(\psi):=\{p \mid \varphi(p)>-\infty\} \neq 0)$. 
The epigraph $\mathcal{E} p(\varphi)$ of an extended function $\varphi$ is the set of pairs $(x, \lambda) \in X \times \mathbb{R}$ such that $\varphi(x) \leq \lambda$ and the hypograph $\mathcal{H} y p(\psi)$ of a function $\psi$ is the set of pairs $(p, \mu) \in X \times \mathbb{R}$ such that $\mu \leq \psi(p)$. Note that the hypograph of $\psi$ is related to the epigraph of $\varphi$ by the relation

$$
(p, \lambda) \in \mathcal{H} y p(\psi) \text { if and only if }(p,-\lambda) \in \mathcal{E} p(\varphi)
$$

An extended function is lower semicontinuous if and only if its epigraph is closed and upper semicontinuous if and only if its hypograph is closed.

Definition 9.9 Hypoderivatives and Superdifferentials. The hypoderivative $D_{\downarrow} \psi(p)$ and the epiderivative $D_{\uparrow} \varphi(p)$ are related to the tangent cones of the hypograph of $\psi$ and epigraph of $\varphi$ by the relations

$$
\mathcal{H} y p\left(D_{\downarrow} \psi(p)\right):=T_{\mathcal{H} y p(\psi)}(p, \psi(p)) \text { and } \mathcal{E} p\left(D_{\uparrow} \varphi(p)\right):=T_{\mathcal{E} p(\varphi)}(p, \varphi(p))
$$

The superdifferential $\partial_{+} \psi(p)$ of the concave function $\psi$ at $p$ is defined by

$$
u \in \partial_{+} \psi(p) \text { if } \forall v \in X,\langle u, v\rangle \geq D_{\downarrow} \psi(p)(v)
$$

and the subdifferential $\partial_{-} \varphi(p)$ is defined by

$$
u \in \partial_{-} \varphi(p) \text { if } \forall v \in X,\langle u, v\rangle \leq D_{\uparrow} \varphi(p)(v)
$$

We infer that

$$
\forall v \in X, \quad D_{\downarrow} \psi(p)(v)=-D_{\uparrow} \varphi(p)(v)
$$

and that

$$
u \in \partial_{+} \psi(p) \text { if and only if } u \in-\partial_{-} \varphi(p)
$$

The polar cone $P^{-}$of a given set $P$ is defined by:

$$
P^{-}=\left\{p \in X^{\star} \mid \forall x \in P,\langle p, x\rangle \leq 0\right\}
$$

where $X^{\star}$ is the dual space of $X$ and the normal cone $N_{K}(x):=T_{K}(x)^{-}$to $K$ at $x \in K$ we use in this paper is the polar cone to the contingent cone to $K$ at $x \in K$. The superdifferential $\partial_{+} \psi(p)$ and the subdifferential $\partial_{-} \varphi(p)$ are related to the normal cones of the hypograph of $\psi$ and epigraph of $\varphi$ by the relations

$$
u \in \partial_{+} \psi(p) \text { if and only if }(-u, 1) \in N_{\mathcal{H} y p(\psi)}(p, \psi(p))
$$

and

$$
u \in \partial_{-} \varphi(p) \text { if and only if }(u,-1) \in N_{\mathcal{E} p(\varphi)}(p, \varphi(p))
$$

Recall the Legendre inversion formula:

$$
u \in-\partial_{+} \psi(p) \text { if and only if } p \in \partial_{-} \varphi^{\star}(u)
$$

and the (decreasing) monotonicity property of superdifferential maps $p \leadsto \partial_{+} \psi(p)$ of a concave function:

$$
\forall u_{i} \in \partial_{+} \psi\left(p_{i}\right), i=1,2,\left\langle u_{1}-u_{2}, p_{1}-p_{2}\right\rangle \leq 0
$$

The subdifferential $\partial_{-} \sigma(K, p)$ of the support function is defined by the support zone $\{u \in$ $K$ such that $\sigma(K, p)=\langle p, u\rangle\}$ of $p$ in $K$. See [6] or [76] for more details.

We shall need the following result on tangent and normal cones to hypographs: 


\section{Lemma 9.10 Normal Cones to Hypographs.}

A. If $\psi: X \mapsto \mathbf{R}_{+} \cup\{-\infty\}$ is an extended function and if $D_{\downarrow} \psi(p)(d p)$ is finite, then, for every $w<\psi(p)$ and every $\mu \in \mathbf{R}$, the pair $(d p, \mu)$ belongs to the contingent cone $T_{\mathcal{H} y p(\psi)}(p, w)$ to the hypograph of $\psi$ at $(p, w)$.

B. Consequently, a pair $(u, \lambda)$ belongs to the normal cone $N_{\mathcal{H} y p(\psi)}(p, w)$ to the hypograph of $\psi$ at $(p, w)$ if and only

1. if $w=\psi(p), \lambda>0$ and $u \in-\lambda \partial_{+} \psi(p)$,

2. if $w \leq \psi(p), \lambda=0$ and $u \in\left(\operatorname{Dom}\left(D_{\downarrow} \psi(p)\right)\right)^{-}$.

C. In particular, if the domain of $D_{\downarrow} \psi(p)$ is dense in $X$, then $(u, \lambda)$ belongs to the normal cone $N_{\mathcal{H} y p(\psi)}(p, w)$ to the epigraph of $\psi$ at $(p, w)$ if and only if $\lambda>0$ and $u \in-\lambda \partial_{+} \psi(p)$. This is the case whenever $\psi$ is Lipschitz around $p$.

\section{Proof}

A. Let $(d p, \lambda)$ belong to $T_{\mathcal{H} y p(\psi)}(p, \psi(p))$. Then we know that there exist sequences $h_{n}>0$ converging to $0, d p_{n}$ converging to $d p$ and $\lambda_{n}$ converging to $\lambda$ such that $\left(p+h_{n} d p_{n}, \psi(p)+h_{n} \lambda_{n}\right)$ belongs to $\mathcal{H} y p(\psi)$. Therefore, for $w<\psi(p)$ and $\mu \in \mathbf{R}$ and $h_{n}$ small enough,

$$
\left(p+h_{n} d p_{n}, w+h_{n} \mu\right)=\left(p+h_{n} d p_{n}, \psi(p)+h_{n} \lambda_{n}\right)+\left(0, w-\psi(p)+h_{n}\left(\mu-\lambda_{n}\right)\right) \in \mathcal{H} y p(\psi)
$$

belongs to the hypograph of $\psi$ because $w-\psi(p)+h_{n}\left(\mu-\lambda_{n}\right) \leq 0$ for $h_{n}$ small enough. Therefore, since $d p_{n} \rightarrow p$ and $\mu_{n}:=\mu \rightarrow \mu$, we infer that $(d p, \mu) \in T_{\mathcal{H} y p(\psi)}(p, w)$.

B. Let us consider now a pair $(u, \lambda)$ belonging to the normal cone $N_{\mathcal{H} y p(\psi)}(p, w):=\left(T_{\mathcal{H} y p(\psi)}(p, w)\right)^{-}$to the epigraph of $\psi$ at $(p, w)$ : Therefore,

$$
\forall(d p, \mu) \in T_{\mathcal{H} y p(\psi)}(p, w),\langle(d p, \mu),(u, \lambda)\rangle=\langle u, d p\rangle+\lambda \mu \leq 0
$$

Examine first the case when $w=\psi(p)$, for which $(d p, \mu) \in T_{\mathcal{H} y p(\psi)}(p, \psi(p))$ if and only if $d p \in \operatorname{Dom}\left(D_{\downarrow} \psi(p)\right)$ and $\mu \leq D_{\downarrow} \psi(p)(d p)$. If $\lambda<0$, we obtain a contradiction because, when $\mu \rightarrow-\infty,\langle u, d p\rangle+\lambda \mu \rightarrow+\infty$. Hence

- either $\lambda>0$, and thus, dividing by $\lambda$ and taking $\mu:=D_{\downarrow} \psi(p)(d p)$, we obtain

$$
\forall d p \in \operatorname{Dom}\left(D_{\downarrow} \psi(p)\right),\left\langle\frac{u}{\lambda}, d p\right\rangle+D_{\downarrow} \psi(p)(d p) \leq 0
$$

which means that $-\frac{u}{\lambda} \in \partial_{+} \psi(p)$

- or $\lambda=0$ and we obtain

$$
\forall d p \in \operatorname{Dom}\left(D_{\downarrow} \psi(p)\right), \quad\langle u, d p\rangle \leq 0
$$

which means that $u \in\left(\operatorname{Dom}\left(D_{\downarrow} \psi(p)\right)\right)^{-}$by definition of the polar cone.

When $w<\psi(p)$, inequalities

$$
\forall(d p, \mu) \in T_{\mathcal{H} y p(\psi)}(p, w), \quad\langle(d p, \mu),(u, \lambda)\rangle=\langle u, d p\rangle+\lambda \mu \leq 0
$$


imply that $\lambda=0$ because by property A: Otherwise, $\lambda \mu$ converges to $+\infty$ when $\mu \rightarrow+\infty$ when $\lambda>0$ and when $\mu \rightarrow-\infty$ when $\lambda<0$ since $\mu$ is allowed to range over $\mathbb{R}$. Therefore $u \in\left(\operatorname{Dom}\left(D_{\downarrow} \psi(p)\right)\right)^{-}$because whenever $d p \in \operatorname{Dom}\left(D_{\downarrow} \psi(p)\right)$ and $\mu \in \mathbb{R}$, then $(d p, \mu) \in T_{\mathcal{H} y p(\psi)}(p, w)$.

C. If the domain $D_{\downarrow} \psi(p)$ is dense in $X$, then the polar cone $\left(\operatorname{Dom}\left(D_{\downarrow} \psi(p)\right)\right)^{-}$is $\{0\}$. Therefore, if $\lambda=0$, then by B. , $u=0$. Aside from this trivial case, the only possibility is thus $\lambda>0$ and $u \in-\lambda \partial_{+} \psi(p)$.

\section{References}

[1] O. Alvarez, E.N. Barron and H. Ishi Hopf-Lax formulas for semicontinuous data. Indiana Univ. Math. J. (48)3: 993-1035, 1999

[2] R. Ansorge. What does the entropy condition mean in traffic flow theory? Transportation Research, 24B(2):133-143, 1990.

[3] J. A. Atwell, J. T. BorggandD, and B. B. King. Reduced order controllers for Burgers' equation with a nonlinear observer. Applied Mathematics and Computational Science, 11(6):1311$1330,2001$.

[4] J.-P. Aubin. Contingent derivatives of set-valued maps and existence of solutions to nonlinear inclusions and differential inclusions. In L. Nachbin, editor, Mathematical Analysis and Applications, Part A, Adv. in Math. Suppl. Stud. 7a, pages 159-229. Academic Press, New York, NY, 1981.

[5] J.-P. Aubin. Viability Theory. Systems and Control: Foundations and Applications. Birkhäuser, Boston, MA, 1991.

[6] J.-P. Aubin. Optima and equilibria. Springer-Verlag, New York, NY, 1993.

[7] J.-P. Aubin. Viability kernels and capture basins of sets under differential inclusions. SIAM Journal of Control and Optimization, 40(3):853-881, 2001.

[8] J.-P. Aubin. Boundary-value problems for systems of first-order partial differential inclusions with constraints. Progress in nonlinear differential equations and their applications, 55:25-60, 2003.

[9] J.-P. Aubin and F. CATTE. Bilateral fixed-point and algebraic properties of viability kernels and capture basins of sets. Set-Valued Analysis, 10:379-416, 2002.

[10] J.-P. Aubin and G. Da Prato. Solutions contingentes de l'équation de la variété centrale. $C$. R. Acad. Sci. Paris Sér. I Math., 311:295-300, 1990.

[11] J.-P. Aubin and G. Da Prato. Contingent solutions to the center manifold equation. Ann. Inst. H. Poincaré Anal. Non Linéaire, 9:13-28, 1992.

[12] J.-P. Aubin and H. Frankowska, Set-Valued Analysis, Birkhäuser Boston, Boston, 1990.

[13] J.-P. Aubin and H. Frankowska. Inclusions aux dérivées partielles gouvernant des contrôles de rétroaction. C. R. Acad. Sci. Paris Sér. I Math., 311:851-856, 1990. 
[14] J.-P. Aubin and H. Frankowska. Systèmes hyperboliques d'inclusions aux dérivées partielles. C. R. Acad. Sci. Paris Sér. I Math., 312:271-276, 1991.

[15] J.-P. Aubin and H. Frankowska. Hyperbolic systems of partial differential inclusions. Ann. Scuola Norm. Sup. Pisa Cl. Sci., 4(18), 1992.

[16] J.-P. Aubin and H. Frankowska. Partial differential inclusions governing feedback controls. $J$. Convex Anal., 2:19-40, 1995.

[17] J.-P. Aubin and H. Frankowska. The viability kernel algorithm for computing value functions of infinite horizon optimal control problems. J. Math. Anal. Appl., 201:555-576, 1996.

[18] J.-P. Aubin and H. Frankowska. Set-valued solutions to the Cauchy problem for hyperbolic systems of partial differential inclusions. NoDEA Nonlinear Differential Equations Appl., 4:149$168,1999$.

[19] J. Baker, A. Armaou, and P. D. Christofides. Nonlinear control of incompressible fluid flow: Application to Burgers' equation and 2d channel flow. Journal of Mathematical Analysis and Applications, 252:230-255, 2000.

[20] A. BAlogh and M. Krstic. Burgers' equation with nonlinear boundary feedback: $H_{1}$ stability, well posedness, and simulation. Mathematical Problems in Engineering, 6:189-200, 2000.

[21] M. Bardi and I. Capuzzo-Dolcetta. Viscosity Solutions of Hamilton-Jacobi-Bellman Equations. Birkhäuser, Boston, MA, 1997.

[22] M. BARDi and L. Evans On Hopf's formulas for solutions of Hamilton-Jacobi equations. Nonlinear Anal., Theory Methods Appl. 8: 1373-1381, 1984.

[23] C. Bardos, A. Y. Leroux, and J. C. Nedelec. First order quasilinear equations with boundary conditions. Communications in partial differential equations, 4(9):1017-1034, 1979.

[24] G. BARLes Solutions de viscosité des équations de Hamilton-Jacobi Springer, Paris 1994.

[25] E. N. Barron and R. Jensen. Semicontinuous viscosity solutions for Hamilton-Jacobi equations with convex Hamiltonians. Comm. Partial Differential Equations, 15:1713-1742, 1990.

[26] E. N. Barron and R. Jensen, Optimal control and semicontiuous viscosity solutions, Proc. Amer. Math. Soc., 113 (1991), pp. 393-402.

[27] E. N. Barron, R. Jensen and W. Liu, Hopf-Lax-type formula for $u_{t}+H(u, D u)=0$. J. Differ. Equations 126, No.1, 48-61, 1996.

[28] A. M. Bayen, R. L. Raffard, and C. Tomlin. Network congestion alleviation using adjoint hybrid control: Application to highways. Hybrid Systems, Computation and Control, Lecture Notes in Computer Science 1790, pages 95-110. Springer Verlag, 2004.

[29] T. R. Bewley. Flow control: new challenges for a new renaissance. Progress in Aerospace Science, 37:21-58, 2001.

[30] E. Bourrel and J.-B. Lesort. Mixing micro and macro representations of traffic flow: a hybrid model based on the LWR theory. In 82nd meeting of the Transportation Research Board, Washington, DC, Jan 2003. 
[31] C. Byrnes and H. Frankowska. Unicité des solutions optimales et absence de chocs pour les équations d'Hamilton-Jacobi-Bellman et de Riccati. Comptes-Rendus de l'Académie des Sciences, 315:427-431, 1992.

[32] C. Byrnes and H. Frankowska. Uniqueness of optimal trajectories and the nonexistence of shocks for Hamilton-Jacobi-Bellman and Riccati partial differential equations. In L. Gorniewicz J. Andres and P. Nistri, editors, Differential Inclusions and Optimal Control, Lecture Notes in Nonlinear Analysis, pages 89-112. J. Schauder Center for Nonlinear Studies, 1998.

[33] C. I. Byrnes, D. S. Gilliam, and V. I. Shubov. Semiglobal stabilization of a boundary controlled viscous Burgers' equation. In Proceedings of the $38^{\text {th }}$ IEEE Conference on Decision and Control, pages 680-681, Phoenix, AZ, Dec. 1999.

[34] P. Cardaliaguet, M. Quincampoix, and P. Saint-Pierre. Set-valued numerical analysis for optimal control and differential games. In M. Bardi, T.E.S. Raghavan, and T. Parthasarathy, editors, Stochastic and Differential Games: Theory and Numerical Methods, Annals of the International Society of Dynamic Games. Birkhäuser, Boston, MA, 1999.

[35] P. Cardaliaguet, M. Quincampoix, and P. Saint-Pierre . Set-valued numerical analysis for optimal control and differential games. In M. Bardi, T.E.S. Raghavan, and T. Parthasarathy, editors, Stochastic and Differential Games: Theory and Numerical Methods, Annals of the International Society of Dynamic Games, pages 177-247. Birkhäuser, 1999.

[36] N. Caroff and H. Frankowska. Optimality and characteristics of Hamilton-Jacobi-Bellman equations. Volume 107 of International Series of Numerical Mathematics, pages 169-180. 1992.

[37] N. Caroff and H. Frankowska. A note on conjugate points and shocks in nonlinear optimal control. Bull. of the Polish Academy of Sciences, 42:115-128, 1994.

[38] N. Caroff and H. Frankowska. Conjugate points and shocks in nonlinear optimal control. Transactions of the AMS, 348:3133-3153, 1996.

[39] P. D. Christofides. Nonlinear and Robust Control of Partial Differential Equation Systems: Methods and Applications to Transport-Reaction Processes. Birkhäuser, Boston, MA, 2001.

[40] M. G. Crandall, L. C. Evans, and P.-L. Lions. Some properties of viscosity solutions of Hamilton-Jacobi equations. Transactions of the American Mathematical Society, 282(2):487-502, 1984.

[41] M. G. Crandall and P.-L. Lions. Viscosity solutions of Hamilton-Jacobi equations. Transactions of the American Mathematical Society, 277(1):1-42, 1983.

[42] C. Daganzo. The cell transmission model: a dynamic representation of highway traffic consistent with the hydrodynamic theory. Transportation Research, 28B(4):269-287, 1994.

[43] C. Daganzo. The cell transmission model, part II: network traffic. Transportation Research, 29B(2):79-93, 1995.

[44] W. B. Dunbar, N. Petit, P. Rouchon, and P. Martin. Boundary control of a nonlinear Stefan problem. In Proceedings of the $42^{\text {nd }}$ IEEE Conference on Decision and Control, pages 1309-1314, Maui, HI, Dec. 2003. 
[45] W. B. Dunbar, N. Petit, P. Rouchon, and P. Martin. Motion planning for a nonlinear Stefan problem. ESAIM: Control, Optimisation andCalculus of Variations, 9:275-296, Feb. 2003.

[46] L. C. Evans. Partial Differential Equations. American Mathematical Society, Providence, RI, 1998.

[47] M. Fliess, J. LÉvine, Ph. Martin, and P. Rouchon. Flatness and defect of nonlinear systems: introductory theory and examples. International Journal of Control, 60(6):1327-1361, 1995.

[48] H. Frankowska, L'équation d'Hamilton-Jacobi contingente, C. R. Acad. Sci. Paris Sér. I Math., 304 (1987), pp. 295-298.

[49] H. Frankowska, Optimal trajectories associated to a solution of contingent Hamilton-Jacobi equations, in Proceedings of the 26th IEEE Conference on Decision and Control, Los Angeles, CA, 1987.

[50] H. Frankowska, Optimal trajectories associated to a solution of contingent Hamilton-Jacobi equations, Appl. Math. Optim., 19 (1989), pp. 291-311.

[51] H. Frankowska, Hamilton-Jacobi equation: Viscosity solutions and generalized gradients, $J$. Math. Anal. Appl., 141 (1989), pp. 21-26.

[52] H. Frankowska, Lower semicontinuous solutions to Hamilton-Jacobi-Bellman equations, in Proceedings of the 30th IEEE Conference on Decision and Control, Brighton, UK, 1991.

[53] H. Frankowska, Lower semicontinuous solutions of Hamilton-Jacobi-Bellman equations, SIAM J. Control Optim., 31 (1993), pp. 257-272.

[54] M. Gugat, M. Herty, A. Klar, and G. Leugering. Optimal control for traffic flow networks. Journal of Optimization theory and Applications, 126(3):589-616, 2005.

[55] A. Jameson. Analysis and design of numerical schemes for gas dynamics 1: Artificial diffusion, upwind biasing, limiters and their effect on accuracy and multigrid convergence. International Journal of Computational Fluid Dynamics, 4:171-218, 1995.

[56] A. Jameson. Analysis and design of numerical schemes for gas dynamics 2: Artificial diffusion and discrete shock structure. International Journal of Computational Fluid Dynamics, 4:1-38, 1995.

[57] Z. Jia, C. Chen, B. Coifman, and P. Varaiya. The PeMS algorithms for accurate, real time estimates of $g$-factors and speeds from single loop detectors. In IEEE Intelligent Transportation Systems Conference Proceedings, pages 536-541, Oakland, CA, Aug. 2001.

[58] T. Kobayashi. Adaptive regulator design of a viscous Burgers' system by boundary control. IMA Journal of Mathematical Control and Information, 18(3):427-437, 2001.

[59] M. Krstic. On global stabilization of Burgers' equation by boundary control. Systems and Control Letters, 37:123-142, 1999.

[60] J.-P. Lebacque and J.-B. Lesort. Macroscopic traffic flow models: a question of order. In Transportation and traffic theory, proceedings of the 14th ISTTT, Jerusalem, Israel, July 1999.

[61] M. J. Lighthill and G. B. Whitham. On kinematic waves. II. A theory of traffic flow on long crowded roads. Proceedings of the Royal Society of London, 229(1178):317-345, 1956. 
[62] P.-L.Lions and J.-C. Rochet. Hopf formula and multitime Hamilton-Jacobi equations. Proc. Am. Math. Soc. 96, 79-84, 1986.

[63] H Ly, K. D. Mease, and E. S. Titi. Distributed and boundary control of the viscous burgers' equation. Numerical Functional Analysis and Optimization, 18(1-2):143-188, 1997.

[64] I. Mitchell. A toolbox of level set methods. http://www.cs.ubc.ca/ mitchell, 2005.

[65] I. Mitchell. Application of Level Set Methods to Control and Reachability Problems in Continuous and Hybrid Systems. PhD thesis, Stanford University, 2000.

[66] I. Mitchell, A M. Bayen, and C. J. Tomlin. A time-dependent Hamilton-Jacobi formulation of reachable sets for continuous dynamic games. IEEE Transactions on Automatic Control, 50(7), pp. 947-957, July 2005.

[67] G. F. Newell. A simplified theory of kinematic waves in highway traffic, part I: general theory. Transporation Research B, 27B(4):281-287, 1993.

[68] G. F. NEwELL. A simplified theory of kinematic waves in highway traffic, part II: queueing at freeway bottlenecks. Transporation Research B, 27B(4):289-303, 1993.

[69] G. F. Newell. A simplified theory of kinematic waves in highway traffic, part III: multidestination flows. Transporation Research B, 27B(4):303-313, 1993.

[70] O. A. Oleinik. On discontinuous solutions of nonlinear differential equations. Uspekhi Mat. Nauk., 12:3-73, 1957. English translation: American Mathematical Society, Ser. 2 No. 26 pp. 95-172, 1963.

[71] M. ÖNDER and E. ÖzBAY. Low dimensional modelling and Dirichlet boundary controller design for burgers equation. International Journal of Control, 77(10):895-906, 2004.

[72] N. Petit. Delay Systems. Flatness in Process Control and Control of some Wave Equations. $\mathrm{PhD}$ thesis, Ecole des Mines de Paris, Paris, France, 2000.

[73] S. Plaskacz and M. Quincampoix Oleinik-Lax formulas and multitime Hamilton-Jacobi systems, to appear.

[74] S. Plaskacz and M. Quincampoix On representation formulas for Hamilton-Jacobi equations related to calculus of variation problems, to appear.

[75] P. I. Richards. Shock waves on the highway. Operations Research, 4(1):42-51, 1956.

[76] R.T. Rockafellar and R. Wets, Variational Analysis, Springer-Verlag, New York, 1997.

[77] P. Saint-Pierre, Approximation of the viability kernel, Appl. Math. Optim., 29 (1994), pp. $187-209$.

[78] P. Saint-Pierre, Approximation of capture basins for hybrid systems, in Hybrid Systems: Computation and Control, Proceedings of the HSCC 2002 Conference, Lecture Notes in Comput. Sci. 2034, Springer-Verlag, New York, 2002.

[79] R. C. Smith and M. A. Demetriou. Research Directions in Distributed Parameter Systems. SIAM, Philadelphia, PA, 2000. 
[80] I. S. Strub and A. M. BAyen. Weak formulation of boundary conditions for scalar conservation laws. To appear in International Journal of Robust and Nonlinear Control, 2006.

[81] J. Yi, H. Lin, L. Alvarez, and R. Horowitz. Stability of macroscopic traffic flow through wavefront expansion. In American Control Conference, pages 1484-1490, Anchorage, AK, May. 2002 .

[82] J. Yi, H. Lin, L. Alvarez, and R. Horowitz. Stability of macroscopic traffic flow modeling through wavefront expansion. Transportation Research Part B, 37:661-679, 2003. 


\section{Contents}

$\begin{array}{lll}1 & \text { Introduction } & 2\end{array}$

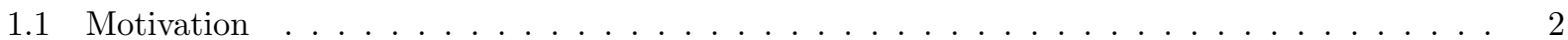

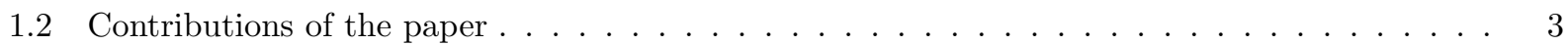

1.3 Outline of the paper $\ldots \ldots \ldots \ldots \ldots \ldots \ldots \ldots$

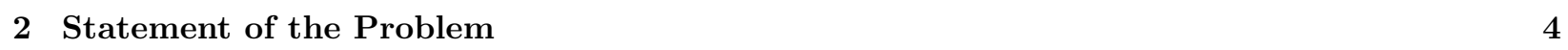

\begin{tabular}{|lrr}
\hline 3 & The Viability Hyposolution & 6
\end{tabular}

\begin{tabular}{|lll}
\hline 4 & The Lax-Hopf formula & 7
\end{tabular}

\begin{tabular}{|lll}
5 & Dirichlet/Initial Conditions and Inequality Constraints & 13
\end{tabular}

\begin{tabular}{|lll}
\hline 6 & Other Auxiliary Systems & 14
\end{tabular}

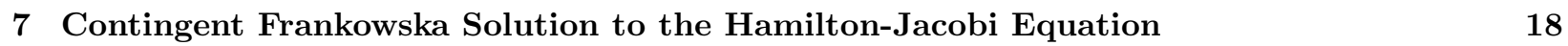

8 Barron-Jensen/Frankowska Solution to the Hamilton-Jacobi Equation 21

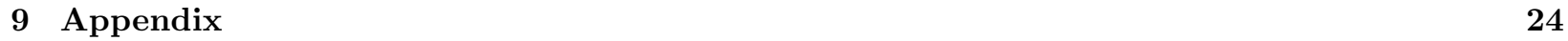

9.1 Some Prerequisites from Viability Theory $\ldots \ldots \ldots \ldots \ldots \ldots \ldots$

9.2 Some Prerequisites of Convex Analysis $\ldots \ldots \ldots \ldots \ldots \ldots \ldots \ldots$ 
Stampato in proprio

MAGGIO 2006

Scuola Normale Superiore, Piazza dei Cavalieri, 7 - PISA 